\title{
POR QUÉ LA GEOGRAFÍA SÍ IMPORTA: FUENTES Y CONSIDERACIONES DE LA INFORMACION AMBIENTAL EN EL PROCESO DEL MODELADO DE NICHOS ECOLÓGICOS Y/O DISTRIBUCIÓN DE ESPECIES
}

\section{WHY GEOGRAPHY MATTERS: SOURCES AND CONSIDERATIONS OF ENVIRONMENTAL INFORMATION IN THE PROCESS OF ECOLOGICAL NICHE AND/OR SPECIES DRISTRIBUTION MODELLING}

\section{CÉSAR A. Ríos-Muñoz ${ }^{1,2}$, Deborah V. Espinosa-MartíneZ ${ }^{3 *}$ Y Katia M. Vega-Flores ${ }^{4}$}

${ }^{1}$ Secretaría de Desarrollo Institucional, Universidad Nacional Autónoma de México, Torre de Rectoría piso 8, Ciudad Universitaria, o4510, Ciudad de México.

${ }^{2}$ UNAM-Costa Rica (Centro de Estudios Mexicanos), edificio del Centro de Investigación y Capacitación en Administración Pública (CICAP), $2{ }^{\circ}$ piso, San Pedro Montes de Oca, 11501-2060 San José, Costa Rica.

${ }^{3}$ Laboratorio de Arqueozoología, Subdirección de Laboratorios y Apoyo Académico, Instituto Nacional de Antropología e Historia, Moneda 16 Centro, 06060, Ciudad de México.

${ }^{4}$ Facultad de Ciencias, Universidad Nacional Autónoma de México, 04510, Ciudad de México.

*Correspondence:dvem@ciencias.unam.mx

Las condiciones o variables ambientales juegan un papel importante en la delimitación de la distribución de las especies, sobre todo cuando nos referimos a escalas regionales, continentalesy/oglobales (Peterson etal., 2011). Estas condiciones son usadas como predictores en el proceso del modelado de nichos ecológicos (MNE) y/o modelado de distribución de especies (MDE), ya que junto con los datos biológicos son los elementos básicos necesarios para su elaboración (Ríos-Muñoz \& Espinosa-Martínez, 2019a, b).

El uso de diferentes variables ambientales recrea la representación del nicho ecológico de las especies, ya que funcionan como parte de las dimensiones del espacio n-dimensional mencionado por Hutchinson (1957). Además, es importante considerar que no todas las variables tienen la misma importancia para todas las especies (Leibold, 1995), por lo que un conocimiento de la historia natural de las especies siempre es importante. Debido a la naturaleza del proceso de MNE/
MDE, las variables ambientales se manejan como coberturas geográficas en formato raster para los sistemas de información geográfica (SIG), por lo que es necesario conocer las fuentes y la calidad de la información. De esta manera, al igual que en la editorial anterior, referente a los datos biológicos (Ríos-Muñoz \& Espinosa-Martinez, 2019a), en esta editorial mencionaremos algunas fuentes y consideraciones de la información ambiental para el proceso de MNE/MDE.

\section{FUENTES DE INFORMACIÓN}

La información ambiental puede provenir de diferentes fuentes, por lo que hay que considerar que es posible clasificarlas dependiendo de su utilidad (e.g. Rodríguez et al., 2007), por su fuente o por las unidades que manejan (Peterson et al., 2011). Por esta razón, hemos clasificado las fuentes con base en el tipo de información que contienen las coberturas, con la finalidad de que puedan ser utilizadas en el proceso de los MNE/MDE o para 
Table 1. Environmental variables classification based on the type of information (detailed sites are shown in appendix 1).

Tabla 1. Clasificación de variables ambientales con base en el tipo de información (los sitios detallados aparecen en el apéndice 1).

\section{Categorías de las variables*}

\section{Climáticas}

2. Pigmentos

3. Salinidad

4. Nutrientes inorgánicos

5. Relieve e hidrología

6. Edafología

7. Uso de suelo, cobertura vegetal, áreas naturales protegidas e incendios

8. Impacto antropogénico

9. Múltiples

\section{Descripción de la categoría}

Contienen datos referentes a las condiciones atmosféricas, terrestres o marinas derivadas de información de precipitación, temperatura, radiación solar y velocidad de viento.

Incluye información sobre el color del océano derivada de la concentración de clorofila.

Se refiere a datos de la proporción de sales disueltas en el agua de mar (e.g. NaCl).

Información relacionada con la cantidad de nutrientes inorgánicos disueltos en el océano (fosfatos, nitratos y silicatos).

Abarca información que describe y/o representa la disposición de la superficie terrestre.

Se refiere a información derivada de las propiedades de los suelos.

Contiene información relacionada con la cobertura vegetal y su dinámica, y los esfuerzos para la conservación de la biodiversidad.

Abarca información que representa el efecto que produce la actividad humana sobre el ambiente.

Se refiere a fuentes de información que contienen más de una categoría previamente descritas.

*El número asignado a cada una de las categorías de las variables corresponde a la numeración presentada en el Apéndice 1.

su análisis posterior de los modelos en un SIG (Tabla 1; Apéndice 1). Vale la pena señalar que no se trata de una clasificación exhaustiva de las variables ambientales y que existen algunas fuentes que son exclusivas para México, como la información contenida en el Portal de Geoinformación del Sistema Nacional de Información sobre Biodiversidad (http://www.conabio.gob. $\mathrm{mx} /$ informacion/gis/) y la infomación del Instituto Nacional de Geografía y Estadística (INEGI, https://www.inegi.org.mx/). Existen diferentes proyectos con información disponible (ver Apéndice 1). Sin embargo, es importante considerar que algunos de estos proyectos pueden estar sujetos a nuevas actualizaciones y, por lo tanto, podrían generar nuevas variables o cambiar de página web.

\section{INFORMACIÓN PROPIA PARA GENERACIÓN DE VARIABLES AMBIENTALES}

Existe la posibilidad de que se disponga de información propia o, sea necesario contar con un tamaño de celda particular que refleje las condiciones a microescala, en cuyo caso será necesario contar con información ambiental del área de estudio. Para poder llevar a cabo el proceso, es necesario tener un conocimiento más allá de la toma de datos de las variables, sobre todo en la forma en la que deben ser interpolados para obtener la resolución que se requiere. Tal es el caso de Contreras et al. (2009) quienes realizaron la toma de datos de 11 variables fisicoquímicas de agua e hicieron la interpolación de dichos datos para generar variables ambientales para poder crear un MDE más preciso de Ambystoma mexicanum.

\section{CONSIDERACIONES EN EL USO DE LA INFORMACIÓN AMBIENTAL}

\section{Datum, elipsoide y sistemas de coordenadas}

Cuando se ocupa información geográfica es necesario considerar algunos aspectos cartográficos como el datum, los elipsoides y los sistemas de coordenadas. El datum es un conjunto de puntos de referencia tomados sobre la superficie terrestre que permite la representación de un modelo de la forma geoide de la Tierra (elipsoide o esferoide) lo más realista posible (Snyder, 1987; Janssen, 2009). El uso de diferentes datums y elipsoides pueden hacer que un mismo punto en la superficie terrestre tenga dos coordenadas diferentes, por lo que se recomienda hacer transformaciones para que el tipo de coordenadas se encuentre con la misma información antes de realizar cualquier análisis (Hager et al., 1990). 
Los sistemas de coordenadas son un sistema de referencia que permite la ubicación espacial. Están basados en tres dimensiones, que en conjunto permiten visualizar, medir y analizar la posición de alguna entidad en, debajo o sobre la superficie terrestre a partir de la posición relativa con respecto al centroide del elipsoide utilizado (Janssen, 2009; Quattrocchi et al., 2017). Existen sistemas de coordenadas no proyectados como el sistema de coordenadas geográficas que se expresa por lo general en sistema sexagesimal (grados, minutos y segundos), o sistemas de coordenadas proyectados (e.g. con tipos de proyección cónica, plana o cilíndrica), que consideran la deformación de la Tierra al representarla en dos dimensiones, tal es el caso del sistema de coordenadas Universal Transversal de Mercator (UTM) que se basa en un sistema de proyección cilíndrica y considera la deformación que existe al aproximarse a los polos creando una representación con base en líneas equidistantes (Deetz \& Adams, 1950; NavarroParraud \& Legorreta-Paulin, 1998). Vale la pena mencionar que para elegir una proyección cartográfica se debe tener en cuenta el área de interés o de estudio, lo que depende de la pregunta de investigación y, por lo tanto, de la ubicación del área en la representación cartográfica (Snyder, 1987).

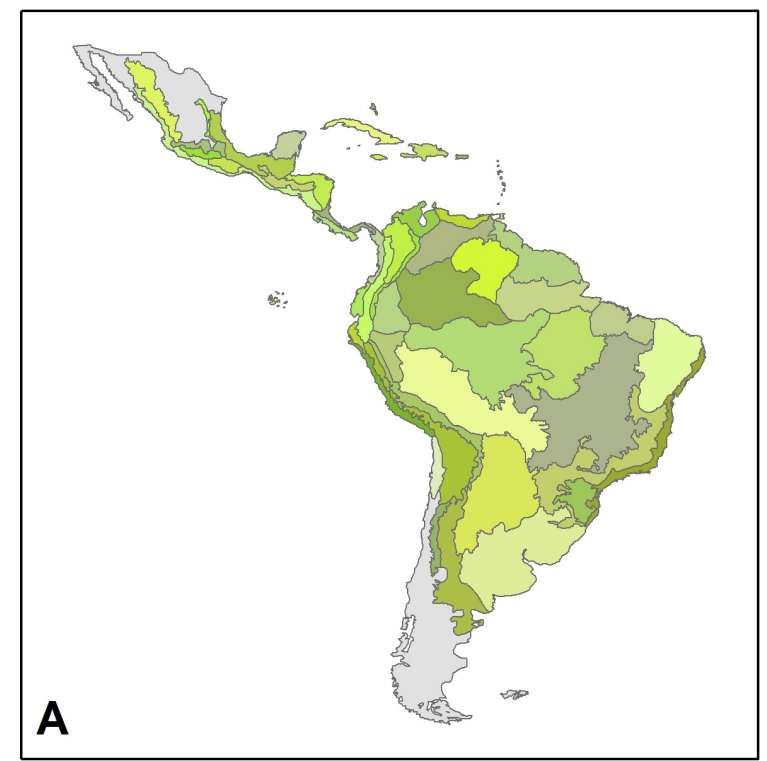

Figure 1. Comparison of formats based on the regionalization of the Neotropics of Morrone (2014). A) Map in vector format of Löwenberg-Neto (2014). B) Raster format converted from vector format with $0.5^{\circ}$ cell size.

Figura 1. Comparación de formatos con base en la regionalización del Neotrópico de Morrone (2014). A) Mapa en formato vector de Löwenberg-Neto (2014). B) Formato raster convertido a partir del formato vector con tamaño de celda de $0.5^{\circ}$.

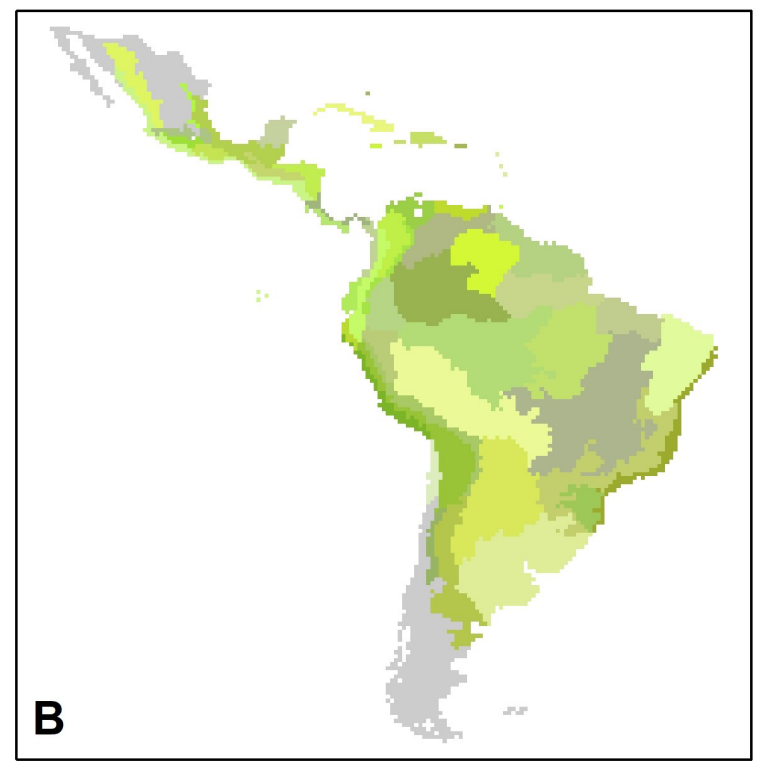

\section{Formatos, resolución espacial y tipo de información}

El manejo de coberturas en un sistema de información geográfica puede hacerse a través de dos tipos en general, formatos vector y raster. El formato vector está formado a partir de puntos, líneas o polígonos que representan la información de interés, mientras que el formato raster está formado por celdas con valores diferenciales que representan la información (NavarroParraud \& Legorreta-Paulin, 1998) (Fig. 1). En el proceso de MNE/MDE se utilizan mapas en formatos raster (e.g. ASCII, BIL en el SIG) aunque es posible utilizar mapas en formato vector transformados al formato raster dependiendo de la información que contengan.

Debido a que los mapas raster son conjuntos de celdas, el tamaño de la celda se convierte en un parámetro importante a considerar, ya que conformará el número de celdas necesarias para representar el área de estudio (Fig. 2). Es necesario considerar que la representación del área de estudio, junto con el tamaño de celda que se escoja, deben coincidir con la resolución espacial del fenómeno biológico que se pretende analizar (Peterson et al., 2011). Esto influye también en la representación que puedan tener los datos biológicos, pues la mayor parte de los algoritmos de modelado consideran la presencia de un registro

B<smiles>CCCC</smiles> 

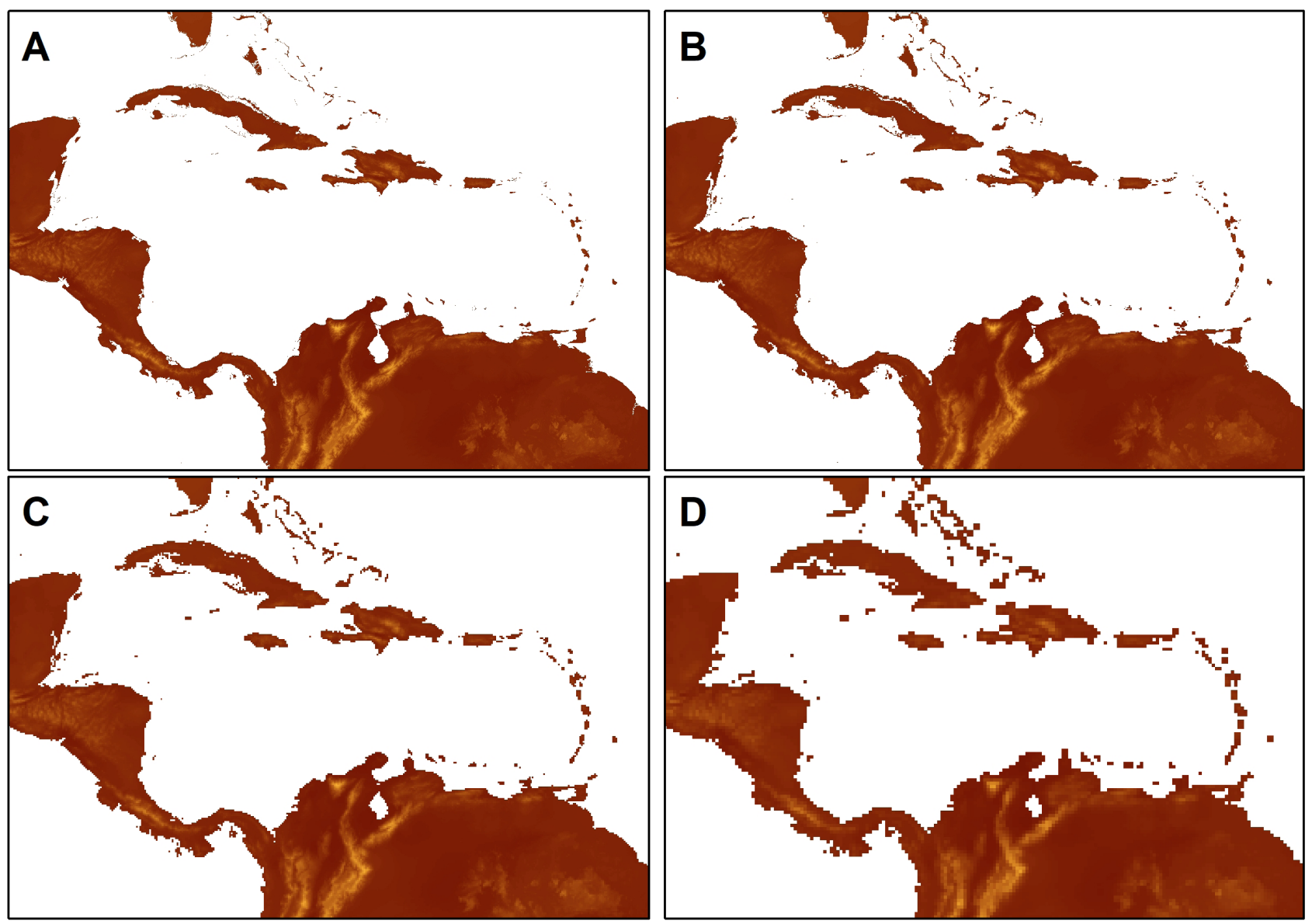

Figure 2. Comparison of layers with different resolution from the same source of information. The layer represents annual mean temperature for the Caribbean region at different cell sizes: A) $30^{\prime \prime}(\sim 1 \mathrm{~km})$, B) $2.5^{\prime}(\sim 5 \mathrm{~km})$, C) $5^{\prime}(\sim 10 \mathrm{~km})$ and D) 10' $(\sim 20 \mathrm{~km})$. Coastlines are totally modified when resolution changes.

Figura 2. Comparación de coberturas con diferente resolución provenientes de la misma fuente de información. La cobertura representa la temperatura media anual para la región del Caribe en donde se representa a diferentes tamaños de celda: A) 30" $\left.(\sim 1 \mathrm{~km}), B) 2.5^{\prime}(\sim 5 \mathrm{~km}), C\right) 5^{\prime}(\sim 10 \mathrm{~km})$ y D) $10^{\prime}(\sim 20 \mathrm{~km})$. La línea costera se modifica por completo al modificar la resolución.

por celda (datos espacialmente únicos en cada pixel) y el uso de tamaños de celda grandes podría hacer que en una celda haya más de un registro, lo que reduciría el número de registros de datos biológicos (Peterson et al., 2011).

La forma de representar la información en las coberturas ambientales también puede ser diferente, por lo que es posible encontrar datos continuos, que son los que tienen unidades de medición como temperatura $\left({ }^{\circ} \mathrm{C}\right)$, precipitación $(\mathrm{mm})$ y altitud (msnm), o datos categóricos, que son representaciones de entidades completas agrupadas bajo una categoría, como la regionalización biogeográfica del Neotrópico (Morrone et al., 2014; digitalizada por Löwenberg-Neto, 2014) (Fig. 1). Algunos algoritmos de modelado tienen la capacidad de trabajar con información categórica (e.g. Maxent), aunque es necesario evaluar si es importante para el análisis que se realizará. En la representación de los datos continuos, existe la posibilidad de tener información que incluya puntos decimales (floating) o que representen números enteros (integer). Esto es esencial saberlo, ya que hay conjuntos de coberturas que usan uno u otro sistema, por ejemplo, las coberturas del proyecto de climatologías a alta resolución para las áreas terrestres de la Tierra (CHELSA por sus siglas en inglés, Karger et al., 2017) tienen el conjunto de coberturas bioclimáticas con valores enteros al haber multiplicado los valores de temperatura original por 10 para evitar el punto decimal, en cambio coberturas como las de 
ecoClimate (Lima-Ribeiro et al., 2015, 2020) presentan valores con decimales.

\section{Calidad de la información}

La calidad de la información ambiental depende por completo de sus fuentes y aunque es posible encontrar las coberturas generadas con los mismos métodos (e.g. variables bioclimáticas de diferentes proyectos, Hijmans et al., 2005; Téllez et al., 2011; Cuervo-Robayo et al., 2014), las fuentes de información y el proceso de depuración puede ser diferente (además de la temporalidad de la información que será tratada más adelante). Por ejemplo, para la cobertura de temperatura media anual se utilizaron 24542 estaciones ya depuradas a nivel mundial para Worldclim 1.4 (Hijmans et al., 2005), mientras que Téllez et al. (2011) utilizó 4262 en un área que va del sur de Estados Unidos al norte de Centroamérica y Cuervo-Robayo et al. (2014) que corresponde a las coberturas más actualizadas para la misma región, utilizaron más de 5000. En este caso la densidad de estaciones meteorológicas también juega un papel importante ya que existen zonas que cuentan con menor cantidad, además de que al limitarse geográficamente, el proceso de depuración de Téllez et al. (2011) y Cuervo-Robayo et al. (2014) fue más preciso que el realizado con una cantidad mayor de datos a nivel mundial (Hijmans et al., 2005), por lo que es importante notar que las diferencias pueden llegar hasta $\pm 10.9^{\circ} \mathrm{C}$ en la temperatura media anual en México (Fig. 3). Es fundamental que los usuarios conozcan los metadatos de las variables a utilizar con la finalidad de que conozcan cómo fueron creadas para que decidan si son útiles o no para los análisis que pretenden realizar (Peterson et al., 2011).

\section{Temporalidad}

La información geográfica, en general, está creada con base en datos primarios que tienen una temporalidad definida. Por esta razón es necesario considerar que no toda la información disponible puede ser utilizada de la misma manera. Existe información de escenarios climáticos desarrollados en tiempo presente con diferentes temporalidades, incluso de una misma fuente de información, por ejemplo los datos bioclimáticos de ecoClimate (Lima-Ribeiro et al., 2015, 2020) incluyen un periodo histórico (1900-1949) y un periodo moderno (1950-1999), mientras que Worldclim en su versión 1.4 incluye datos de $\sim 1960-1990$ (Hijmans et al., 2005) y en su versión 2.1 tiene datos de 1970 al 2000 (Fick \& Hijmans, 2017). Estas diferencias no sólo existen en grandes grupos de datos climatológicos presentes, sino que pueden existir en escenarios temporales de cambio climático ya sea al futuro o al pasado (e.g. reconstrucciones paleoclimáticas) y que han sido utilizados para proyectar el nicho ecológico de las especies bajo las condiciones actuales (e.g. Ochoa-Ochoa et al.,
2012; Cruz-Silva et al., 2012). Algunas fuentes de información también cuentan con datos mensuales que incluso han sido utilizados para identificar modificaciones en la distribución a lo largo del año (Encarnación-Luévano et al., 2011). La correspondencia temporal entre las coberturas ambientales y los datos biológicos es fundamental para una interpretación adecuada del modelo y va también a depender del objetivo de estudio (Peterson et al., 2011).

\section{RECOMENDACIONES ACERCA DE LA INFORMACIÓN AMBIENTAL}

Existen diferentes recomendaciones en el uso de la información ambiental de acuerdo con distintos autores. Algunas de estas recomendaciones son evitar el uso de variables redundantes y considerar la existencia de la autocorrelación espacial como un posible sesgo. Algunos autores han descartado variables debido a que generan anomalías espaciales (Escobar et al., 2014) y otros han reportado que el uso de variables redundantes tiene poco impacto en la representación de los MNE/MDE, a pesar de esto, sugieren cuantificar y reportar el grado de redundancia de las variables (Feng et al., 2019).

\section{Identificación de variables correlacionadas}

Uno de los métodos que ha sido utilizado para evitar información redundante, ha sido eliminar las variables correlacionadas con base en el uso del coeficientes de correlación (e.g. Spearman, Pearson) directamente sobre las variables ambientales como una forma de aproximarse a la identificación de la colinealidad entre las variables (Dormann et al., 2013). Varios trabajos han utilizado diferentes valores del coeficiente de correlación de Pearson como umbral, en general, considerando que las variables con valores $>0.75$ (Rissler \& Apodaca, 2007) y $>0.85$ (Elith et al., 2006, 2010; Sánchez-Ramos et al., 2018) representan información correlacionada. La tendencia general es escoger sólo una variable del conjunto que esté por arriba de ese umbral (Fig. 4). Es importante mencionar, que no existe una regla para determinar el valor umbral, por lo que queda al criterio del investigador.

\section{Identificación de variables multicolineales}

Otra forma que ha sido utilizada es el uso de pruebas de multicolinealidad, como el Factor de Inflación de Varianza (Variance Inflation Factor, VIF) (Naimi et al., 2014). El VIF es calculado con base en el cuadrado del coeficiente de correlación múltiple entre las variables y se ha interpretado que valores VIF $>10$ presentan una señal de colinealidad (Dormann et al., 2013; Naimi et al., 2014; Tytar et al., 2015). Al utilizar diferentes variables, se ha optado por realizar el VIF en una forma gradual 

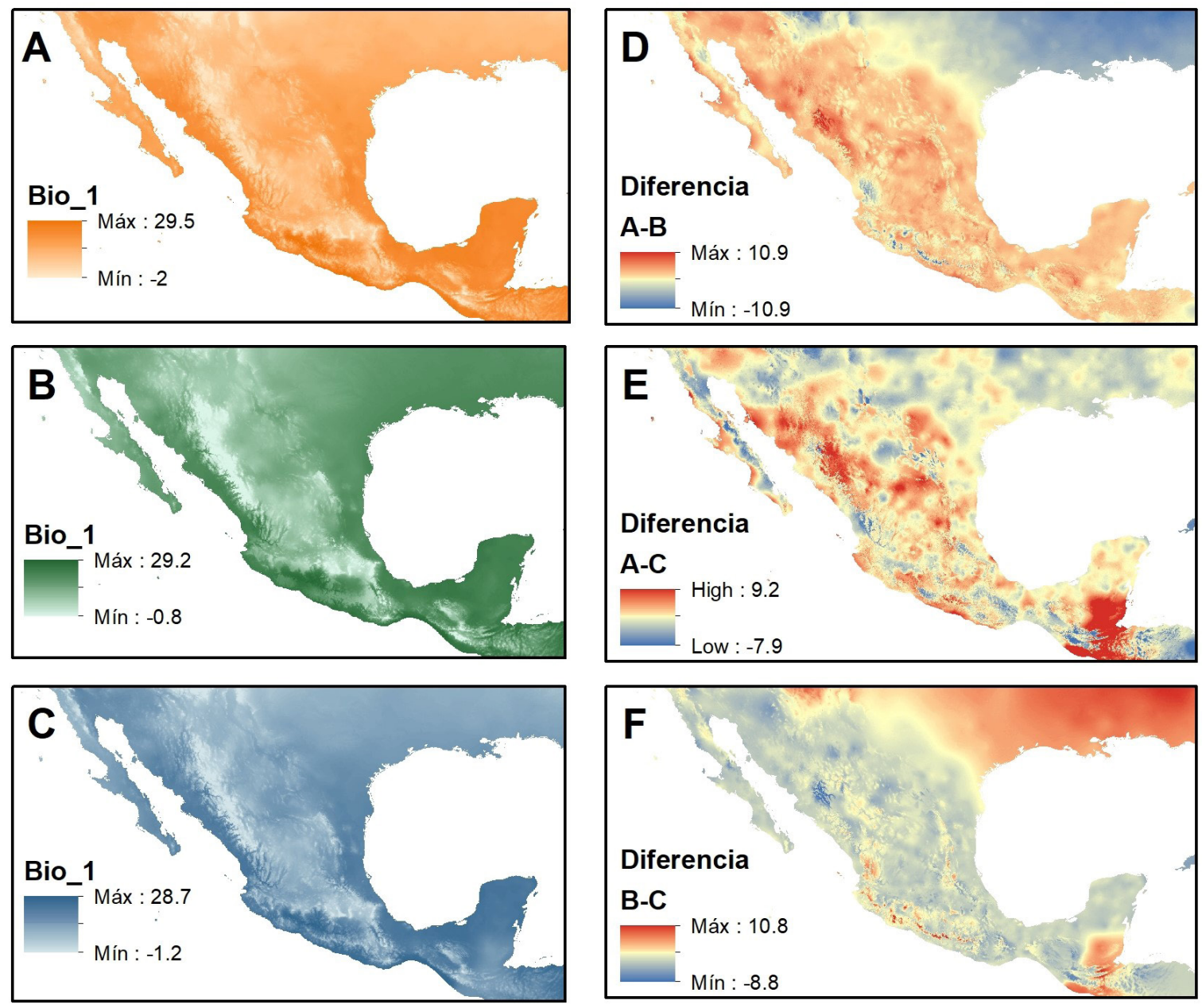

Figure 3. Comparison of the annual mean temperature layers (Bio 1) for Mexico from different sources. A) Worldclim 1.4 (Hijmans et al., 2005). B) Téllez et al. (2011). C) Cuervo-Robayo et al. (2014). E) Difference between WorldClim 1.4 and Téllez et al. (2011), F) Difference between Worldclim 1.4 and Cuervo-Robayo et al. (2014). G) Difference between Téllez et al. (2011) y Cuervo-Robayo et al. (2014). Maximum and minimum values represent ${ }^{\circ} \mathrm{C}$. Even if they represent the same layer there are differences up to $\pm 10.9^{\circ} \mathrm{C}$ due to the sources of information and the cleaning process of the data.

Figura 3. Comparación de coberturas de temperatura media anual (Bio 1) para México de diferentes fuentes. A) Worldclim 1.4 (Hijmans et al., 2005). B) Téllez et al. (2011). C) Cuervo-Robayo et al. (2014). E) Diferencia entre WorldClim 1.4 y Téllez et al. (2011), F) Diferencia entre Worldclim 1.4 y Cuervo-Robayo et al. (2014). G) Diferencia entre Téllez et al. (2011) y Cuervo-Robayo et al. (2014). Los valores máximos y mínimos representan ${ }^{\circ} \mathrm{C}$. A pesar de que se trata de la misma cobertura existen diferencias de hasta $\pm 10.9^{\circ} \mathrm{C}$ debido a las fuentes de información y al proceso de limpieza de los datos.

o iterativa para excluir todas las variables con valores de VIF > 10 (Naimi et al., 2014). Aunque este valor es arbitrario, ha sido utilizado como una forma de evitar variables con información redundante.

\section{Análisis de componentes principales de las variables}

El análisis de componentes principales (PCA por su siglas en inglés) es un método multivariado que transforma un conjunto de variables correlacionadas en nuevas variables (componentes) no correlacionadas (Janžekovič \& Novak, 2012). Esto asegura la independencia de las variables utilizadas en el proceso de modelado, ya que cada componente sintetiza la máxima variabilidad residual contenida en las coberturas originales, además de que se cuantifica el grado de contribución que aporta cada variable original (loading values) (Pla \& de Miranda, 1986; Peterson et al., 2011). Algunos autores han utilizado el PCA como una forma de contar con variables no correlacionadas para el proceso de $\mathrm{MNE} / \mathrm{MDE}$, aunque la interpretación de 

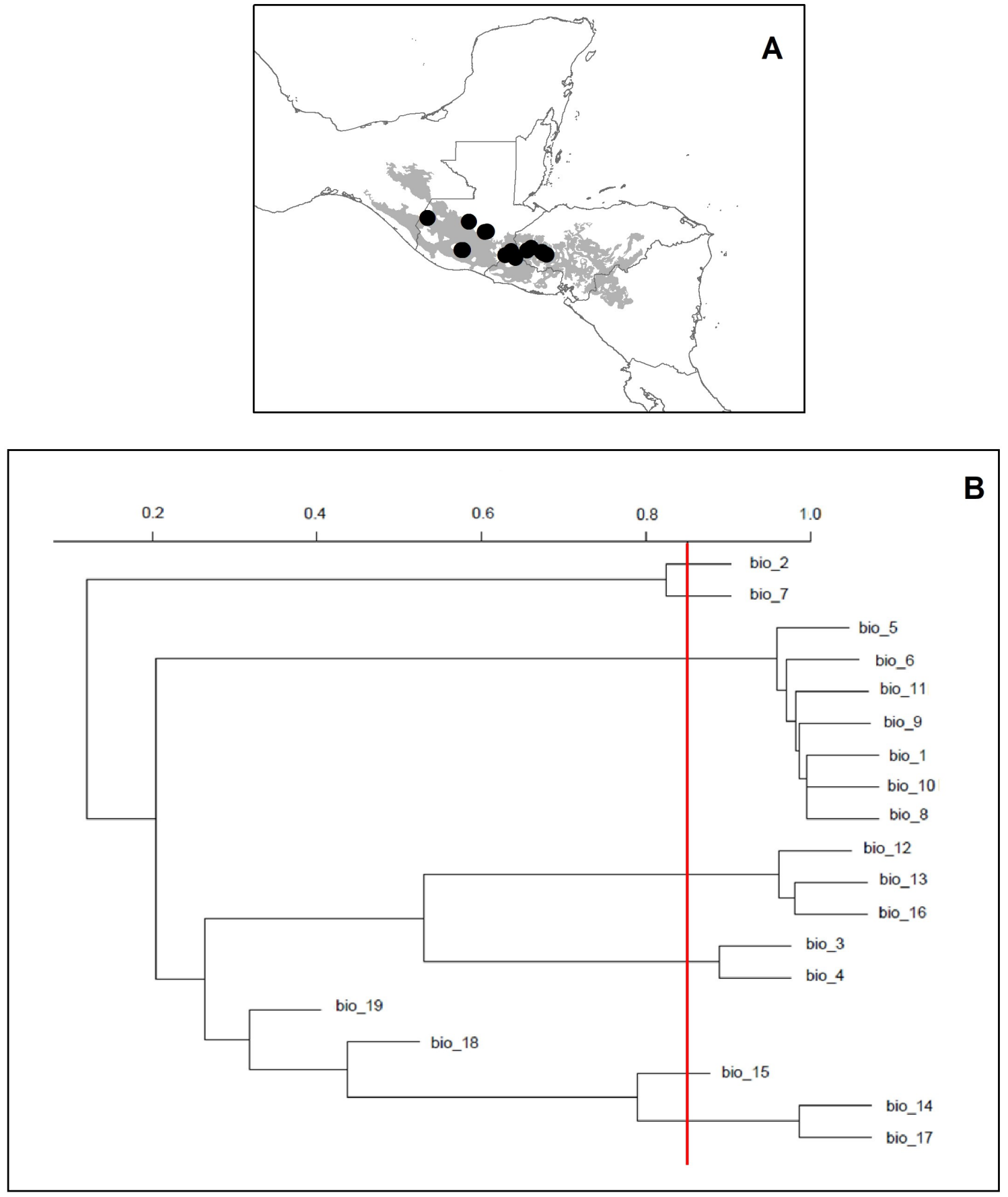

Figure 4. Correlation analysis for the ecoregions of Incilius ibarrai. A) Black dots indicate the presence records of Incilius ibarrai, in grey area marked the ecoregions corresponding to the area considered for the correlation analysis. B) Phenogram of UPGMA based on the Pearson correlation coefficient, for the 19 bioclimatic layers for the ecoregions where Incilius ibarrai is present. The red line is the threshold of 0.85 , values above represent variables highly correlated.

Figura 4. Análisis de correlación para las ecorregiones de Incilius ibarrai. A) Los puntos negros indican registros de presencia de Incilius ibarrai, en gris se marcan las ecorregiones que corresponden al área que se tomará en cuenta para hacer el análisis de correlación. B) Fenograma de UPGMA con base en el coeficiente de correlación de Pearson de 19 variables bioclimáticas para las ecorregiones con presencia de Incilius ibarrai. La línea roja es un valor umbral de 0.85 los valores por encima de esta representan variables con alta correlación. 
los componentes no es tan sencilla, incluso hay trabajos en los que los aportes de cada variable han sido utilizados para poder analizar coberturas en otros escenarios temporales (e.g. YañezArenas et al., 2016). Es importante mencionar que si las variables originales no están correlacionadas el PCA no ofrece ninguna ventaja (Pla \& de Miranda, 1986).

\section{Autocorrelación espacial}

Es necesario tener en cuenta que así como las variables ambientales pueden contener información redundante, se ha visto que los datos biológicos (registros de las especies) y las variables ambientales presentan autocorrelación espacial (Sokal \& Oden, 1978a, b; Legendre, 1993; Griffith, 1996, 2003; Rossi \& Quénéhervé, 1998; Diniz-Filho et al., 2003; Veloz, 2009; de Oliveira et al., 2014). La autocorrelación espacial está definida como la correlación que existe entre los valores de una sola variable debido a la proximidad que éstos presentan en el espacio geográfico, esto quiere decir, que existe dependencia entre los valores de una variable en ubicaciones o localidades que son vecinas, es por ello que una variable tomará valores similares cuando las localidades se encuentren a una distancia corta (autocorrelación positiva) y valores diferentes a distancias largas (autocorrelación negativa) (Legendre, 1993; Griffith, 1996, 2003). También se ha observado que se pueden tener autocorrelaciones positivas con datos que presentan distancias largas o negativas en distancias cortas, las cuales se deben a la representación geográfica de la información ambiental (Legendre \& Fortin, 1989; Rossi \& Quénéhervé, 1998).

Para determinar la autocorrelación espacial de los datos, se han utilizado diferentes métodos. Uno de los más utilizados es el Coeficiente I de Moran (CM), el cual implica un cálculo de los productos cruzados de los valores de la media ajustada a los vecinos geográficos, es decir, covariación. Los valores que se calculan con el CM varían entre -1 a 1, que indican independencia o similitud total de los datos respectivamente, los valores que sean iguales a 0 indican un patrón espacial aleatorio (Griffith, 1996, 2003). Para hacer el cálculo de la autocorrelación espacial de una serie de datos, es posible generar varias matrices de distancias geográficas y obtener los valores de CM para una misma variable, lo que permite evaluar el comportamiento de la autocorrelación en función de la distancia espacial y generar un correlograma espacial en la que se observa el patrón espacial de los datos (Diniz-Filho et al., 2003). La utilización de este coeficiente no sólo se ha empleado para determinar la distancia en la que los datos dejan de presentar autocorrelación espacial en análisis geográficos, sino que también se ha utilizado para señalar cómo, la corrección de la autocorrelación puede mejorar la precisión de los MNE/MDE y actuar de manera diferente en el espacio geográfico y en el ecológico (de Oliveira et al., 2014). Incluso, se ha utilizado junto con otros métodos (e.g. prueba de Mantel) en estudios en los que se combinan los MNE con datos de diversidad genética, para observar el efecto que puede tener la autocorrelación espacial en procesos ecológicos y evolutivos (Diniz-Filho et al., 2016).

Otra forma con la cual se ha evaluado el efecto que tiene la autocorrelación espacial a la hora de realizar MNE/MDE, es haciendo un filtrado espacial de los datos y posteriormente comparando los resultados obtenidos. Este filtrado se puede hacer de diferentes maneras, una opción es la propuesta por Veloz (2009), quien parte de datos únicamente de presencias y evalúa las distancias en las que los datos dejan de ser similares a partir del cálculo de semivariogramas, los cuales modelan la varianza entre pares de puntos en función de la distancia a la cual la semivarianza alcanza un umbral (intervalo) que indica la independencia espacial de los datos. Otra forma, es filtrando los datos a partir de la eliminación al azar de algunas localidades que se encuentren a una distancia determinada a priori considerando la heterogeneidad geográfica (e.g. zonas montañosas) que presente el área de estudio o a partir de la implementación de un generador de matrices de distancia geográfica (Boria et al., 2014).

Debido a la necesidad que actualmente se tiene de obtener un MNE/MDE que no presente un ajuste a los datos de inicio como resultado de la presencia de autocorrelación espacial, algunos autores han diseñado herramientas enfocadas en el tratamiento de los datos de entrada para la obtención de MNE/MDE. Muscarella et al. (2014) desarrollaron un paquete en R llamado ENMeval el cual crea un conjunto de datos para la validación cruzada de $\mathrm{K}$ iteraciones utilizando diferentes métodos para particionar los datos de ocurrencia, posteriormente, se construye una serie de modelos candidatos usando Maxent con una serie de configuraciones determinadas por el usuario y por último, se proporcionan diversas medidas de evaluación que permitan seleccionar la configuración óptima del modelo. Posteriormente, Aiello-Lammens et al. (2015) proponen que es necesario realizar un adelgazamiento de los datos de entrada a partir de un método que permitan reducir el efecto que tiene el sesgo de muestreo en el MDE/MDE y no solo hacerlo de manera manual, por lo que desarrollan un paquete en $\mathrm{R}$ llamado spThin, el cual genera un conjunto de datos que presenta el número máximo de registros para una distancia de adelgazamiento dada, cuando se ejecuta una serie suficiente de iteraciones. 


\section{Evaluación de las variables ambientales}

Recientemente se ha recomendado hacer un análisis de selección de la información ambiental con la finalidad de seleccionar el mejor conjunto de variables que puede estar asociado con los datos biológicos, teniendo un mejor resultado en el proceso de modelado (Cobos et al., 2019). Sin duda, la evaluación de las variables se ha hecho mediante procesos heurísticos como una forma de descartar aquellas que no representen información para la especie. El proceso que proponen Cobos et al. (2019) es un proceso exhaustivo en el que se consideran las variables y el conjunto de datos biológicos con el algoritmo Maxent. El proceso consiste en generar diferentes modelos con los diferentes conjuntos de coberturas y parámetros para el algoritmo para que pueda ser evaluado por medio del Criterio de Información de Akaike corregido para muestras pequeñas (AICc por sus siglas en inglés). Aunque representa una manera novedosa de evaluar las variables ambientales y los datos biológicos, han habido críticas para su utilización ya que los modelos con valores más bajos de AICc presentan valores altos en errores omisión y comisión (Velasco \& González-Salazar, 2019) .

Así como la información biológica es fundamental para el proceso de modelado, la información ambiental también lo es. Es por ello, que es necesario considerar que existen factores que pueden alterar el resultado de los MNE/MDE. Además, que es importante detenerse a pensar qué es lo que se requiere, qué es lo más conveniente y considerar cómo debe de usarse toda esta información, evitando así el uso de procesos automatizados sólo porque están disponibles.

Agradecimientos.- A la Dra. Leticia M. Ochoa Ochoa por su invitación para participar en esta serie de editoriales sobre MNE/MDE. A Salvador Hernández Rubio por su ayuda con la información de la distribución de Incilius ibarrai.

\section{LITERATURA CITADA}

Aiello-Lammens, M.E., R.A. Boria, A. Radosavljevic, B. Vilela \& R.P. Anderson. 2015. spThin: a R package for spatial thinning of species occurrence records for use in ecological niche models. Ecography 38:541-545.

Boria, R.A., L.E. Olson, S.M. Goodman \& R.P. Anderson. 2014. Spatial filtering to reduce sampling bias can improve the performance of ecological niche models. Ecological Modelling 275:73-77.

Cobos, M.E., A.T. Peterson, L. Osorio-Olvera \& D. Jiménez-García. 2019. An exhaustive analysis of heuristic methods for variable selection in ecological niche modeling and species distribution modeling. Ecological Informatics 53:100983.

Contreras, V., E. Martínez-Meyer, E. Valiente \& L. Zambrano. 2009. Recent decline and potential distribution in the last remnant area of the microendemic Mexican axolotl (Ambystoma mexicanum). Biological Conservation 142:2881-2885.

Cruz-Silva, J.A. 2012. Reconstrucción paleoambiental del Pleistoceno final usando modelos de nicho ecológico inferidos con vertebrados: el caso de la cueva de San Josecito, Nuevo León. Tesis de Maestría. Posgrado en Ciencias Biológicas, Universidad Nacional Autónoma de México, México.

Cuervo-Robayo, A.P., O. Téllez-Valdés, M.A. Gómez-Albores, C.S. Venegas-Barrera, J. Manjarrez \& E. Martínez-Meyer. 2014. An update of high-resolution monthly climate surfaces for Mexico. International Journal of Climatology 34:2427-2437.

de Oliveira, G., T.F. Rangel, M.S. Lima-Ribeiro, L.C. Terribile \& J.A.F. Diniz-Filho. 2014. Evaluating, partitioning, and mapping the spatial autocorrelation component in ecological niche modeling: a new approach based on environmentally equidistant records. Ecography 37:637-647.

Deetz, C.H. \& O.S. Adams. 1950. Elementos de proyección de mapas y su aplicación a la construcción de mapas y cartas. Government Print office, Washington, D.C., USA.

Diniz-Filho, J.A.F., L.M. Bini \& B.A. Hawkins. 2003. Spatial autocorrelation and red herrings in geographical ecology. Global Ecology \& Biogeography 12:53-64.

Diniz-Filho, J.A.F., A.C.O.F. Barbosa, R.G. Collevatti, L.J. Chaves, L.C. Terribile. M.S. Lima-Ribeiro \& M.P.C. Telles. 2016. Spatial autocorrelation analysis and ecological niche modelling allows inference of range dynamics driving the population genetic structure of a Neotropical savanna tree. Journal of Biogeography 43:167-177.

Dormann, C.F., J. Elith, S. Bacher, C. Buchmann, G. Carl, G. Carré, J.R.G. Marquéz, B. Gruber, B. Lafourcade, P.J. Leitão, T. Münkemüller, C. McClean, P.E. Osborne, B. Reineking, B. Schröder, A.K. Skidmore, D. Zurell \& S. Lautenbach. 2013. Collinearity: a review of methods to deal with it and a simulation study evaluating their performance. Ecography 36:27-46.

Elith, J., C.H. Graham, R.P. Anderson, M. Dudik, S. Ferrier, A. Guisan, R.J. Hijmans, F. Huettmann, J.R. Leathwick, A. Lehmann, 
J. Li, L.G. Lohmann, B.A. Loiselle, G. Manion, C. Moritz, M. Nakamura, Y. Nakazawa, J.M.M. Overton, A.T. Peterson, S.J. Phillips, K. Richardson, R. Scachetti-Pereira, R.E. Schapire, J. Soberon, S. Williams, M.S. Wisz \& N.E. Zimmermann. 2006. Novel methods improve prediction of species' distributions from occurrence data. Ecography 29:129-151.

Elith, J., M. Kearney \& S. Phillips. 2010. The art of modelling rangeshifting species. Methods in Ecology and Evolution 1:330-342.

Encarnación-Luévano, A., O.R. Rojas-Soto \& J.J. Sigala-Rodríguez. 2013. Activity response to climate seasonality in species with fossorial habits: A niche modeling approach using the lowland burrowing treefrog (Smilisca fodiens). PLoS ONE 8:e78290.

Escobar, L.E.,A. Lira-Noriega, G. Medina-Vogel\&A.T. Peterson. 2014. Potential for spread of the white-nose fungus (Pseudogymnoascus destructans) in the Americas: use of Maxent and NicheA to assure strict model transference. Geospatial Health 9:221-229.

Feng, X., D.S. Park, Y. Liang, R. Pandey \& M. Papeş. 2019. Collinearity in ecological niche modeling: Confusions and challenges. Ecology and Evolution 9:10365-10376.

Fick, S.E. \& R.J. Hijmans. 2017. WorldClim 2: new $1 \mathrm{~km}$ spatial resolution climate surfaces for global land areas. International Journal of Climatology 37:4302-4315.

Griffith, D.A. 1996. Spatial autocorrelation and eigenfunctions of the geographic weights matrix accompanying geo-referenced data. The Canadian Geographer 40:351-367.

Griffith, D.A. 2003. Spatial autocorrelation and spatial filtering: gaining understanding through theory and scientific visualization. Springer-Verlag, Berlín, Alemania.

Hager, J.W., F.L. Larry, S.S. Jacks \& D.R. Hill. 1990. Datums, Ellipsoids, Grids, and Grid Reference Systems. Defense Mapping Agency, Washington, D.C., USA.

Hijmans, R.J., S.E. Cameron, J.L. Parra, P.G. Jones \& A. Jarvis. 2005 Very high resolution interpolated climate surfaces for global land areas. International Journal of Climatology 25:1965-1978.

Hutchinson, G.E. 1957. Concluding remarks. Cold Spring Harbor Symposia on Quantitative Biology 22:415-427.

Janssen, V. 2009. Understanding Coordinate Systems, Datums and Transformations in Australia. Pp. 697-715. En B. Ostendorf, P.
Baldock, D. Bruce, M. Burdett \& P. Corcoran (Eds.), Proceedings of the Surveying \& Spatial Sciences Institute Biennial International Conference 2009, Surveying and Spatial Sciences Institute, Adelaide, Australia.

Janžekovič, F. \& T. Novak. 2012. PCA-A Powerful Method for Analyze Ecological Niches. Pp. 127-142. En P. Sanguansat (Ed.), Principal Component Analysis: Multidisciplinary Applications. InTech. Rijeka, Croacia.

Karger, D.N., O. Conrad, J. Böhner, T. Kawohl, H. Kreft, R.W. Soria-Auza, N.E. Zimmermann, H.P. Linder \& M. Kessler. 2017. Climatologies at high resolution for the earth's land surface areas. Scientific Data 4:170122.

Legendre, P. 1993. Spatial autocorrelation: trouble or new paradigm? Ecology 74:1659-1673.

Legendre, P. y M.J. Fortin. 1989. Spatial pattern and ecological analysis. Vegetatio 80:107-138.

Leibold, M.A. 1995. The Niche concept revisited: mechanistic models and community context. Ecology 76:1371-1382.

Lima-Ribeiro, M.S., S. Varela, J. González-Hernández, G. de Oliveira, J.A.F. Diniz-Filho \& L.C. Terribile. 2015. ecoClimate: a database of climate data from multiple models for past, present, and future for Macroecologists and Biogeographers. Biodiversity Informatics 10:1-21.

Lima-Ribeiro, M.S., S. Varela, J. González-Hernández, G. de Oliveira, J.A.F. Diniz-Filho, A.T. Peterson \& L.C. Terribile. 2020. The ecoClimate Database. http://ecoclimate.org, [Consultado en Abril 2020].

Löwenberg-Neto, P. 2014. Neotropical region: a shapefile of Morrone's (2014) biogeographical regionalisation. Zootaxa 3802:300.

Morrone, J.J. 2014. Biogeographical regionalisation of the Neotropical region. Zootaxa 3782:1-110.

Muscarella, R., P.J. Galante, M. Soley-Guardia, R.A. Boria, J.M. Kass, M. Uriarte \& R.P. Anderson. 2014. ENMeval: An R package for conducting spatially independent evaluations and estimating optimal model complexity for MAXENT ecological niche models. Methods in Ecology and Evolution 5:1198-1205. 
Naimi, B., N.A.S. Hamm, T.A. Groen, A.K. Skidmore \& A.G. Toxopeus. 2014. Where is positional uncertainty a problem for species distribution modelling? Ecography 37:191-203.

Navarro Parraud, M.d.C. \& G. Legorreta Paulin. 1998. Sistemas de Información Geográfica: Teoría introductoria y ejercicios con AutoCAD e IDRISI. Publicaciones Docentes del Museo de Zoología "Alfonso L. Herrera", Facultad de Ciencias, UNAM, D.F., México.

Ochoa-Ochoa, L.M., P. Rodríguez, F. Mora, O. Flores-Villela \& R.J. Whittaker. 2012. Climate change and amphibian diversity patterns in Mexico. Biological Conservation 150:94-102.

Peterson, A.T., J. Soberón, R.G. Pearson, R.P. Anderson, E. MartínezMeyer, M. Nakamura \& M.B. Araujo. 2011. Ecological niches and geographic distributions. Princeton University Press, New Jersey, USA.

Pla, L.E. \& F. de Miranda. 1986. Análisis multivariado: método de componentes principales. Secretaría General de la Organización de Estados Americanos, Washington, D.C., USA.

Quattrocchi, D.A., E.A. Wentz, N. Siu-Ngan Lam \& C.W. Emerson. 2017. Integrating scale in remote sensing and GIS. CRC Press, Boca Raton, Florida, USA.

Ríos-Muñoz, C.A. \& D.V. Espinosa-Martínez. 2019a. Datos biológicos: fuentes y consideraciones. Revista Latinoamericana de Herpetología 2:5-14.

Ríos-Muñoz, C.A. \& D.V. Espinosa-Martínez. 2019b. ¿Qué es necesario considerar para utilizar de manera adecuada los modelos de nicho ecológico o modelos de distribución de especies?: Cómo no caer en la tentación. Revista Latinoamericana de Herpetología 2:5-8.

Rissler, L.J. \& J.J. Apodaca. 2007. Adding more ecology into species delimitation: Ecological niche models and phylogeography help define cryptic species in the Black Salamander (Aneides flavipunctatus). Systematic Biology 56:924-942.

Rodríguez, J.P., L. Brotons, J. Bustamante \& J. Seoane. 2007. The application of predictive modelling of species distribution to biodiversity conservation. Diversity and Distributions 13:243251.
Rossi, J.P. \& P. Quénéhervé. 1998. Relating species density to environmental variables in presence spatial autocorrelation: a study case on soil nematodes distribution. Ecography 21:117-123.

Sánchez-Ramos, L.E., A. Gordillo-Martínez, C.R. GutiérrezArellano, T. Kobelkowsky-Vidrio, C.A. Ríos-Muñoz \& A.G. Navarro-Sigüenza. 2018. Bird diversity patterns in the Nuclear Central American Highlands: a conservation priority in the northern Neotropics. Tropical Conservation Science 11:1-17.

Snyder, J.P. 1987. Map Projections-A working manual. U.S. Geological Survey Professional Paper 1395. Washington, D.C., USA.

Sokal, R.R. \& N.L. Oden. 1978a. Spatial autocorrelation in biology. 1. Methodology. Biological Journal of Linnean Society 10:199-228.

Sokal, R.R. \& N.L. Oden. 1978b. Spatial autocorrelation in biology. 2. Some biological implications and four applications of evolutionary and ecological interest. Biological Journal of Linnean Society 10:229-249.

Téllez, O., M.A. Hutchinson, H.A. Nix \& P. Jones. 2011. Desarrollo de coberturas digitales climáticas para México. Pp. 67-70. En G. Sánchez Rojas, C. Ballesteros Barrera \& N.P. Pavón (Eds.), Cambio Climático. Aproximaciones para el estudio de su efecto en la biodiversidad. Universidad Autónoma del Estado de Hidalgo, Pachuca, Hidalgo, México.

Tytar, V., S. Mezhzherin \& L. Sobolenko. 2015. Using ecological niche modeling for biodiversity conservation guidance in the Western Podillya (Ukraine): Amphibians. Vestnik Zoologii 49:135-144.

Velasco, J.A. \& C. González-Salazar. 2019. Akaike information criterion should not be a "test" of geographical prediction accuracy in ecological niche modelling. Ecological Informatics 51:25-32.

Veloz, S.D. 2009. Spatially autocorrelated sampling falsely inflates measures of accuracy for presence-only niche models. Journal of Biogeography 36:2290-2299.

Yañez-Arenas, C., A.T. Peterson, K. Rodríguez-Medina \& N. Barve. 2016. Mapping current and future potential snakebite risk in the new world. Climatic Change 134:697-711. 


\title{
APÉNDICE 1. FUENTES DE INFORMACIÓN AMBIENTAL DE ACUERDO CON LA CLASIFICACIÓN PROPUESTA
}

\author{
César A. Ríos-Muñoz, Deborah V. Espinosa-Martínez y Katia M. Vega-Flores
}

Recopilación de fuentes basada en una compilación propia más referencias de Rodríguez et al. (2007), la base de datos de variables de Cuervo-Robayo (2019, Marzo 22) y de Emetere \& Akinlabi (2020).

\section{ABREVIATURAS COMUNES}

CMIP: Proyecto de inter-comparación de modelos de clima acoplados (Coupled Model Intercomparison Project).

CRU-TS: Series temporales de la Unidad de Investigación Climática (Climatic Research Unit Timeseries).

GCM: Modelos generales climáticos (General climatic models).

IPCC: Panel Internacional de Cambio Climático (Intergovernmental Panel on Climate Change).

MODIS: Espectroradiómetro de Imágenes de Resolución Moderada (Moderate-Resolution Imaging Spectroradiometer).

PMIP: Proyecto de Intercomparación de Modelos Paleoclimáticos (Paleoclimate Modelling Intercomparison Project).

RCP: Trayectorias de concentración representativas (Representative Concentration Pathways).

SRTM: Misión Topográfica Shuttle Radar (Shuttle Radar Topography Mission).

SST: Temperatura Superficial del Océano (Sea Surface Temperature).

UMG: Último máximo glacial.

\section{CLIMÁTICAS}

1.1 AdaptWest: Current and projected climate data for North America (https://adaptwest.databasin.org/pages/adaptwest-climatena). Incluye 27 variables bioclimáticas y 48 variables mensuales para los periodos 1961-1990 y 1981-2010, además de escenarios de predicción para 2020, 2050, 2080 en dos RCP de ocho GCM para Norteamérica (Wang et al., 2016).
1.2 Center for Environmental Data Analysis (CEDA): Climatic Research Units Time-Series (CRU TS) v3.26 (https://catalogue.ceda.ac.uk/ uuid/7ad889f2cc1647efba7e6a356098e4f3?). Comprende variables con variaciones climáticas mensuales (1901-2017), con una escala casi global (excluyendo la Antártida) y una resolución espacial de $0.5^{\circ}$ (Harris et al., 2014; Harris \& Jones, 2019).

1.3 Climate Hazards Group InfraRed Precipitation with Station data (CHIRPS): Rainfall Estimates from Rain Gauge and Satellite Observations v2.0 (https://www.chc.ucsb.edu/data/chirps). Incluye variables relacionadas a las estimaciones de precipitación a partir del uso de pluviómetros y observaciones satelitales (1981-presente), con una escala casi global y una resolución de $0.05^{\circ}$ (Funk et al., 2014).

1.4 ClimateSA: historical and projected climate data for Mexico, Central and South America v1.12 (https://sites.ualberta.ca/ ahamann/ data/climatesa.html). Software que permite estimar más de 50 variables mensuales, estacionales o anuales a partir de datos CRU-TS 3.22 desde 1901 actualizados a 2013. Datos de proyecciones futuras están basados en 15 GCM del conjunto de datos del multimodelo CMIP5 correspondiente al IPCC5 para las RCP de los años 2020, 2050 y 2080. La información se encuentra disponible para México, Centro y Sudamérica (Hamann et al., 2013).

1.5 Climatologies at high resolution for the Earth's land surface areas (CHELSA) v1.2 (http://chelsa-climate.org/). Incluye variables climáticas (precipitación y temperatura) mensuales para la superficie terrestre (1979-2013), con una escala casi global $\left(84^{\circ} \mathrm{N}\right.$ a 90 S) y una resolución espacial de 30" (Karger et al., 2017).

1.6 CliMond: Global climatologies for bioclimatic modelling v1.2 (https:// www.climond.org/). Incluye 35 variables bioclimáticas del periodo 1961-1990 y escenarios futuros (IPCC5) incluyendo varios GCMs para el área terrestre al 2030, 2050, 2070, 2080, 2090 y 2100, con una escala casi global (excluyendo la Antártida) y una resolución espacial de 10' y 30' (Kriticos et al., 2012). 
1.7 Daily Surface Weather Data on a 1-km Grid for North America (Daymet) v3.4 (https://daac.ornl.gov/cgi-bin/dataset_lister. $\mathrm{pl}$ ? $\mathrm{p}=32$ ). Incluye estimaciones de parámetros meteorológicos diarios, mensuales y anuales desde 1980 hasta el año completo más reciente para Norteamérica (incluido Puerto Rico y Hawái), con una resolución espacial de $1 \mathrm{~km}^{2}$ (Thornton et al., 2016a, b, c).

1.8 Downscaled and debiased climate simulations for North America from 21,000 years ago to 21000AD (https://datadryad.org/stash/ dataset/doi:10.5061/dryad.1597g). Incluye información de simulaciones climáticas para Norteamérica desde hace 21000 años (UMG) hasta proyecciones futuras hasta el año 2100 , con una resolución de $0.5^{\circ}$ (Lorenz et al., 2016a, b).

1.9 ecoClimate (https://www.ecoclimate.org/). Incluye variables climáticas mensuales (precipitación y temperatura) basadas en los modelos climáticos CMIP5 y PMIP3. Se incluyen proyecciones para el presente ( 1760,1900-1949, 1950-1999), el pasado(Plioceno, Holoceno medio y UMG) y para el futuro (2080-2100). Para las proyecciones a futuro se incluyen cuatro escenarios (RCP 2.6, RCP 4.5, RCP 6.0 y RCP 8.5). Todos presentan una escala global y una resolución de $0.5^{\circ}$ (Lima-Ribeiro et al., 2015, 2020).

1.10 Ecospat - Spatial Ecology Group: Bioclim variables for Southern South America (https://www.unil.ch/ecospat/home/menuguid/ ecospat-resources/data.html). Incluye 19 variables bioclimáticas para el periodo 1950-2000 para el Sur de Sudamérica (incluyendo Chile, Bolivia, Perú y Argentina), con una resolución espacial de 1 $\mathrm{km}$ (Pliscoff et al., 2014).

1.11Global Integrated Drought Monitoring and Prediction System(GIDMaPS) Data Sets (https://figshare.com/collections/Global_Integrated_ Drought_Monitoring_and_Prediction_System_GIDMaPS_ Data_Sets/853801). Abarca variables de múltiples indicadores de sequía, derivada de datos de precipitación y humedad del suelo. Este conjunto de datos incluyen datos históricos y predicciones probabilísticas de sequías de diferentes fuentes y diferentes periodos (1980-presente, 1979-presente, 1949-presente), basados en una escala casi global y una resolución de $2 / 3^{\circ} \times 1 / 2^{\circ}, 0.125^{\circ}$, $0.5^{\circ}, 2.5^{\circ} \mathrm{y}^{\circ}$ (Hao et al., 2014).

1.12 Group for High Resolution Sea Surface (GHRSST) Level 4 MUR Global Foundation Sea Surface Temperature Analysis v4.1 (https://podaac. jpl.nasa.gov/dataset/MUR-JPL-L4-GLOB-V4.1). Contiene variables que describen la SST para el periodo 2002-presente, con una escala global y una resolución espacial de $1 \mathrm{~km}^{2}$. El análisis L4 Multiscale Ultrahigh Resolution (MUR) está basado en observaciones nocturnas de SST y GHRSST L2P de varios instrumentos: MODIS, AMSRE, entre otros (JPL MUR MEaSUREs Project, 2015).

1.13 Hadley Centre Sea Ice and Sea Surface Temperature (HadISST) v1.1 (https://climatedataguide.ucar.edu/climate-data/sst-datahadisst-vi1). Engloba variables climáticas (SST y cobertura de hielo marino) para el periodo 1870-2015, con una escala global y una resolución de $1^{\circ}$. Los datos incluidos en este portal se derivaron del Research Data Archive (Hadley Centre for Climate Prediction and Research, 2006).

1.14 Köppen-Geiger climate classification maps (http://www. gloh2o.org/koppen/). Incluye variables climáticas basadas en la clasificación de Köppen-Geiger para las condiciones históricas actuales (1980-2016) y condiciones climáticas proyectadas hacia el futuro (2071-2100), con una escala global y una resolución espacial de $1 \mathrm{~km}^{2}$ (Beck et al., 2018).

1.15 MERRAclim v2 (https://datadryad.org/stash/dataset/ doi:10.5061/dryad.s2v81). Incluye 19 variables bioclimáticas de las décadas de los 1980's, 1990's, 2000's. Son obtenidas a partir de datos de temperatura y humedad máxima, media y mínima. Tienen escala global con una resolución 10', 5' y 2.5' (Vega et al., 2017, 2018).

1.16 microClim: Global estimates of hourly microclimate based on longterm monthly climate averages (https://figshare.com/collections/ microclim_Global_estimates_of_hourly_microclimate_based_ on_long_term_monthly_climate_averages/878253). Incluye estimaciones de las condiciones microclimáticas a nivel mensual para el periodo 1961-1990, con una escala casi global (no considera las zonas polares) y una resolución espacial de $\sim 15 \mathrm{~km}$ (Kearney et al., 2014).

1.17 PaleoClim (http://www.paleoclim.org/). Incluye 19 variables bioclimáticas proyectadas hacia el pasado (Pleistoceno y Plioceno) y para el futuro (2080-2100), con una escala global y una resolución espacial de $30^{\prime \prime}, 2.5^{\prime}, 5^{\prime}$ y 10'. Las variables se derivan de los valores mensuales de temperatura y precipitación (Brown et al., 2018).

1.18 Satellite-based time-series of sea-surface temperature since 1981 for climate applications (SST CCI) v2 (https://springernature. figshare.com/articles/Metadata_record_for_Satellite-based time-series_of_sea-surface_temperature_since_1981_for climate_applications/9939638). Incluye variables climáticas que describen la SST para el periodo de 1981-2016, con una escala global y una resolución de $0.05^{\circ}$ (Merchant et al., 2019a, b). 
1.19 Superficies climáticas para México (http://idrisi.uaemex.mx/ distribucion/superficies-climaticas-para-mexico, https://drive. google.com/drive/u/o/folders/1AZWY8TtXSdrBI5g4ZELuRZU MXPOCmiUU?fbclid=IwAR03Ziuhmtc84DEk5QW5tDziGkLXd 39170VLNFctWVsTwAwVC39JXVmypdY). Incluye 19 variables bioclimáticas para el periodo 1910-2009 para México (sur de Estados Unidos al norte de Centroamérica), con una resolución espacial $\sim 1 \mathrm{~km}$ y $90 \mathrm{~m}$ (Cuervo-Robayo et al., 2014).

1.20 TerraClimate (http://www.climatologylab.org/terraclimate. html). Contiene dos conjuntos de datos: 1) datos de clima primarios (temperatura máxima y mínima, presión de vapor, precipitación acumulada, radiación de onda corta superficial, velocidad de viento), y 2) Variables derivadas (Evapotranspiración de referencia de Penman-Montieth, escorrentía, evapotranspiración actual, déficit climático de agua, humedad del suelo, equivalencia de nieve y agua, índice de severidad de sequía de Palmer, déficit de presión de vapor). La información corresponde al periodo 1958-2015, con una escala global y una resolución espacial de $0.04^{\circ}$ (Abatzoglou et al., 2018).

1.21 WorldClim (https://worldclim.org). Existen dos versiones: 1.4 y 2.1 por la diferencia en la temporalidad de los datos disponibles (1960-1990 y 1970-2000). La versión 1.4 (no incluye la Antártida) incluye coberturas de temperatura, precipitación y 19 variables bioclimáticas, además de coberturas de escenarios de cambio climático con datos del CMIP5. Las coberturas a futuro son las proyecciones climáticas del IPCC $5_{5}$ de los modelos climáticos globales para cuatro RCPs para el 2050 y 2070. Las coberturas paleoclimáticas para el Holoceno medio son de nueve GCMs, tres para el UMG y uno para el último interglacial, estos dos últimos para el Pleistoceno. La versión 2.1 tiene una escala global e incluye la información de temperatura, precipitación (mensual y agrupada para el periodo), radiación solar, velocidad de viento y presión de vapor de agua, las 19 coberturas bioclimáticas y elevación. Además, incluye escenarios de cambio climático a futuro con base en datos del CMIP6 de nueve modelos climáticos globales para los periodos 2021-2040, 2041-2060, 2061-2080, 2081-2100 y cuatro trayectorias socioeconómicas compartidas. También se incluyen los datos mensuales para cada periodo de 20 años. Ambas versiones están disponibles con diferentes resoluciones espaciales 30", 2.5', 5' y 10' (Hijmans et al., 2005; Fick \& Hijmans, 2017).

\section{PIGMENTOS}

2.1 NASA Ocean Color (https://oceancolor.gsfc.nasa.gov/). Contiene información sobre el color del océano, la cual es obtenida por el
Ocean Biology Processing Group (OBPG) en el NASA's Goddard Space Flight Center a partir de información satelital de diferentes misiones: a) SeeWifs lanzado por Orbital Sciences Corporation en el satélite OrbView-2 o SeaStar que recopiló información desde septiembre de 1997 hasta finales de la misión en diciembre de 2010, y que presentan una resolución de $4 \mathrm{~km}$ a nivel global y de $1 \mathrm{~km}$ a nivel local; b) MODIS-Aqua y MODIS-Terra MODIS es un instrumento que se colocó en dos satélites Terra (EOS AM) y Aqua (EOS PM) los cuales realizan un recorrido alrededor de la Tierra cada dos días de norte a sur a través del Ecuador por la mañana y de sur a norte por el Ecuador en la tarde respectivamente, los datos obtenidos juegan un papel importante en la comprensión de la dinámica global y los procesos que ocurren en tierra, océanos y atmósfera inferior; c) VIIRS-SNPP es un instrumento que se encuentra en una serie de naves espaciales que conforman el Sistema Conjunto de Satélites Polares (JPSS por sus siglas en inglés) incluida la Asociación Nacional de Orbital Polar Suomi (SNPP por sus siglas en inglés), VIIRS es el sucesor de MODIS para la generación de datos a partir de 22 bandas espectrales (16 de resolución moderada [750 $\mathrm{m}$ en el nadir], cinco bandas de resolución de imagen [375 m] y una banda día-noche [DNM]), los datos cubren un periodo desde el 2 de enero de 2012 al presente; d) OCTS es un instrumento que se encuentra a bordo del Satélite Avanzado de Observación de la Tierra (ADEOS por sus siglas en inglés) lanzado por la Agencia Espacial Japonesa (NASDANational Space Development Agency) y cuenta con 12 bandas que cubren las regiones visibles, infrarrojo cercano e infrarrojo térmico, con un ancho de banda de $1400 \mathrm{~km}$ aproximadamente y una resolución nadir de $700 \mathrm{~m}$; e) CZCS es el escáner de color de la zona costera y fue el primer instrumento dedicado a la medición del color del océano y volado en una nave espacial, presentó seis bandas espectrales de las cuales cinco se utilizan principalmente para el color del océano, cubre un periodo desde el 30 de octubre de 1978 hasta el 22 de junio de 1986 (NASA-GSFC-OBPG, 2014).

\section{SALINIDAD}

3.1 NASA EOSDIS, Physical Oceanography Distributed Active Archive Center (PO.DAAC): JPL SMAP Level 3 CAP Sea Surface Salinity Standard Mapped Image 8-Day Running Mean V4.3 Validated Dataset (https:// podaac.jpl.nasa.gov/dataset/SMAP_JPL_L3_SSS_CAP_8DAYRUNNINGMEAN_V43). Contiene información de salinidad a nivel superficial del mar con una cobertura a nivel global, una resolución temporal de ocho días desde el 31 de marzo 2015 al presente, generada en el Observatorio NASA Soil Moisture Active Passive (SMAP). Presenta una resolución de $0.6^{\circ}$ (Fore et al., 2016; JPL, 2020). 


\section{NUTRIENTES INORGÁNICOS}

4.1 Nutrient profiles vertical distribution (https://resources.marine. copernicus.eu/?option=com_csw\&task=results?option=com csw\&view=details\&product_id=MULTIOBS_GLO_BIO NUTRIENTS_PROFILES_REP_015_009). Perfiles verticales marinos de concentración de nitratos, fosfatos y silicatos desde la superficie hasta los $8000 \mathrm{~m}$ de profundidad a nivel global. El cálculo se realiza para cada flotador Argo equipado con un sensor de oxígeno, aplicando un método llamado sistema de carbonato y concentración de nutrientes de las propiedades hidrológicas y oxígeno usando una red neuronal (CANYON por sus siglas en inglés) considerando la información de los últimos 30 años de la base de datos GLODAPv2 (https://www.glodap.info/) (Sauzede et al., 2017; Bittig et al., 2018).

\section{RELIEVE E HIDROLOGÍA}

5.1 ETOP01 Global Relief Model (https://www.ngdc.noaa.gov/mgg/ global/). Se trata de un modelo de relieve de la Tierra, que incluye topografía y batimetría. Puede descargarse con la información de las capas de hielo en los polos o sin ellas. Ambas están disponibles con una resolución de I' (Amante \& Eakins, 2009).

5.2 EMODnet-Bathymetry (https://www.emodnet-bathymetry.eu/ data-products). Es un modelo digital batimétrico que cubre las regiones marítimas de Europa (límites $90^{\circ} \mathrm{N}, 36^{\circ} \mathrm{E}$ y $15^{\circ} \mathrm{N} 43^{\circ} \mathrm{E}$ ) y que se generó a partir de datos de levantamientos batimétricos, modelos digitales de elevación compuestos, información batimétrica derivada de datos satelitales. Los datos presentan una resolución de 1/16' (EMODnet Bathymetry Consortium, 2018).

5.3 General Bathymetric Chart of the Oceans (GEBC0_2019): Gridded Bathymetry Data (https://www.gebco.net/data_and_products/ gridded_bathymetry_data/). Incluye un modelo digital batimétrico. Es un modelo general de terreno global para tierra y océano con una escala global y una resolución espacial de 15 ". La cuadrícula de este modelo usa como base la versión I del conjunto de datos SRTM15_plus y se basa en la versión 11 de SRTM30_plus. GEBCO_2019 consiste en un proyecto que tiene como objetivo recopilar todos los datos batimétricos disponibles para generar un mapa para el fondo oceánico global para el 2030 (GEBCO, 2019).

5.4 Geomorpho90m- Global high- resolution geomorphometry layers: empirical evaluation and accuracy assessment (https://peerj.com/ preprints/27595/). Incluye variables que representan diferentes características de geomorfometría derivadas del Modelo de
Elevación Digital de el Terreno Mejorado con Eliminación de Errores múltiples (MERIT-DEM), con la finalidad de representar las variaciones topográficas a escala global, con una resolución de 3" y 7.5" (Amatulli et al., 2019).

5.5 Global Islands Explorer (GIE) (https://rmgsc.cr.usgs.gov/gie/). Incluye un mapa con formato vector de línea de costa global que contiene tres clases de islas: continental, islas de más de $1 \mathrm{~km}^{2}$ e islas de menos de $1 \mathrm{~km}^{2}$. Los datos se obtuvieron a partir de imágenes satelitales Landsat de 2014 y se encuentran disponibles a una escala global y una resolución espacial de $30 \mathrm{~m}$ (Sayre et al., 2019).

5.6 Global Multi-resolution Terrain Elevation Data 2010 (GMTED2010) (https://www.usgs.gov/centers/eros/science/usgs-eros-archivedigital-elevation-global-multi-resolution-terrain-elevation?qtscience_center_objects=0\#qt-science_center_objects). Contiene siete coberturas de elevación con una escala terrestre global y una resolución espacial de 30", 15" y 7.5" (Danielson \& Gesch, 2011).

5.7 Global River Widths from Landsat (GRWL) (https://zenodo.org/ record/1297434\#.XqMu8MgzY2x). Incluye una base de datos de la geometría de los ríos (ancho y largo) a escala casi global (excluyendo la Antártida). Los datos se obtuvieron a partir de la recopilación de datos de 3693 estaciones de calibración y 7376 imágenes de Landsat TM (Thematic Mapper), ETM+ (Enhanced Thematic Mapper Plus) y OLI (Operational Land Imager) (Allen \& Pavelsky, 2018).

5.8 Hydrological data and maps based on Shuttle Elevation Derivatives at multiple Scales (HydroSHEDS) (https://hydrosheds.cr.usgs.gov/ index.php). Contiene información hidrográfica que incluye modelo digital de elevación, cuencas, red de ríos, dirección y acumulación de flujo. Los datos están disponibles a escala regional y casi global con una resolución espacial de 3", 15" y 30 " (Lehner et al., 2008).

5.9 International Satellite Land Surface Climatology Project Initiative II (ISLSCP II): HYDROlk Elevation-derived Products (https://daac. ornl.gov/cgi-bin/dsviewer.pl?ds_id=1007). Incluye parámetros generados a partir de datos de elevación. Los datos se encuentran disponibles a escala global y una resolución espacial de $1^{\circ}$ y $0.5^{\circ}$ (Verdin et al., 2011).

5.10 SRTM 90m DEM Digital Elevation Database v4.1 (https://cgiarcsi. community/data/srtm-90m-digital-elevation-database-V4-1/). Modelo digital de elevación con una resolución de $90 \mathrm{~m}$. Todos los datos se pueden descargar como mosaicos de $5^{\circ} \times 5^{\circ}$ (Jarvis 
et al., 2008). En la página se incluyen ligas de algunos modelos digitales de elevación auxiliares de algunos países específicos.

\section{EDAFOLOGÍA}

6.1 Interpolation of Mexican soil properties at a scale of 1:1,000,000 (https://www.sciencedirect.com/science/article/abs/pii/ So016706113002504). Incluye nueve capas de la distribución de algunas propiedades del suelo $(\mathrm{Ca}, \mathrm{K}, \mathrm{Mg}, \mathrm{Na}, \mathrm{C}$ orgánico, materia orgánica, conductividad eléctrica, relación de absorción de sodio y pH) generadas a partir de 4400 muestras de suelo tomadas en territorio mexicano, con una resolución espacial de 1 km (Cruz-Cárdenas et al., 2014).

6.2 Soil Grids - global gridded soil information (https://www.isric. org/explore/soilgrids). Incluye modelos predictivos de siete profundidades estándar $(0,5,15,30,60,100$ y $200 \mathrm{~cm})$ para algunas propiedades del suelo, con una escala global y una resolución espacial de $250 \mathrm{~m}$. Estos modelos se obtuvieron a partir de observaciones de perfil de suelo de la base de datos WoSIS y una serie de covariables ambientales (más de 400 capas ambientales) (e.g. clima, cobertura del suelo y morfología del terreno) (Hengl et al., 2017).

6.3 World Soil Information Service (WoSIS) (https://www.isric. org/explore/wosis). Incluye datos de 196498 perfiles de suelo (las propiedades químicas y físicas del suelo, así como su clasificación: FAO, WRB y USDA) procedentes de 173 países, obteniendo aproximadamente un total de 832 mil capas del suelo $u$ horizontes. Los datos obtenidos fueron recopilados de diferentes proveedores de datos (Batjes et al., 2017, 2020).

\section{USO DE SUELO, COBERTURA VEGETAL, ECORREGIONES, ÁREAS NATURALES PROTEGIDAS E INCENDIOS}

7.1 CCI-LC (http://maps.elie.ucl.ac.be/CCI/viewer/). Cuenta con mapas de cobertura de suelo con una resolución espacial de 300 $\mathrm{m}$, con una temporalidad que va de 1992 hasta 2015 (Defourny et al., 2012; Bontemps et al., 2013, 2015; Li et al., 2016, 2018).

7.2 CLUMondo (https://github.com/VUEG/CLUMondo). Está basado en la aproximación del sistema terrestre, que son sistemas socioecológicos que reflejan el uso de suelo en unidades espaciales en términos de la composición de la cobertura de suelo, configuración espacial y manejo de actividades empleadas. En contraste a las clasificaciones de cobertura de suelo, el papel de la intensidad del uso de suelo y los sistemas pecuarios son explícitos (van Vliet \& Verburg, 2017).

REVISTA LATINOAMERICANA DE HERPETOLOGÍA Vol.03 №.01 / Mayo 2020
7.3 Comisión Nacional de Áreas Naturales Protegidas (México) (http:// sig.conanp.gob.mx/website/pagsig/info_shape.htm). Incluye mapas en formato vector de las áreas naturales protegidas hasta julio 2019, zonificación primaria hasta julio 2019, regiones CONANP hasta mayo 2017, y áreas destinadas voluntariamente a la conservación para México (CONANP, 2020).

7.4 Esfuerzos sociales de Conservación (no certificados) (https://drive.google.com/drive/folders / I EuY Yo8v7t8AYouIgc4vqlNqXOaLErmp?usp=sharing). Conjunto de bases de datos que incluyen: ordenamientos comunitarios territoriales y reservas privadas para el territorio mexicano (Bezaury-Creel et al., 2012).

7.5 Forest Observation System (FOS) (https://www.forestobservation-system.net/). Iniciativa internacional que tiene como objetivo establecer una base de datos de biomasa forestal in situ a escala casi global (excepto la Antártida) (Schepaschenko et al., 2019).

7.6 Global 1-degree Maps of Forest Area, Carbon Stocks, and Biomass, 1950-2010 (https://daac.ornl.gov/VEGETATION/guides/Global_ Biomass_1950-2010.html). Contiene información sobre el área forestal a nivel mundial, reservorios de carbono y biomasa forestal con una resolución de $1^{\circ}$ y una temporalidad de 1950 al 2010 (Hengeveld et al., 2015).

7.7 Global Fire Emissions Database (GFED) v4 (https://www. globalfiredata.org/). Incluye información relacionada con incendios y emisiones de carbono, gases traza y aerosoles (1997-presente), con una escala global y una resolución de $0.25^{\circ}$. Los datos se obtuvieron a partir de la combinación de información satelital sobre la actividad del fuego y la productividad de la vegetación para estimar el área quemada por mes y las emisiones de los incendios (Randerson et al., 2018).

7.8 Global Land Analysis \& Discovery (https://glad.umd.edu/). Es un laboratorio del Departamento de Ciencias Geográficas de la Universidad de Maryland. Dentro de los datos que se encuentran disponibles se hallan: a) Global 2010 Tree Cover (30 m) (https://glad. umd.edu/dataset/global-2010-tree-cover-30-m) que incluye mapas que representan la extensión, pérdida y ganancia de la cobertura arbórea para el periodo 2000 a 2012 . Los datos están disponibles a escala casi global $\left(80^{\circ} \mathrm{N} \mathrm{a} 60^{\circ} \mathrm{S}\right)$, con una resolución espacial de $30 \mathrm{~m}$ (Hansen et al., 2013); b) Global land change from 1982 to 2016 (https://glad.umd.edu/dataset/long-term-globalland-change) cuenta con mapas que presentan cambios totales en la cobertura vegetal, suelo sin vegetación y cambio de uso de suelo para el periodo 1982-2016. Los datos se obtuvieron a partir 
de observaciones satelitales diarias a partir del Radiómetro Avanzado de muy Alta Resolución (AVHRR por sus siglas en inglés) y están disponibles a escala global, con una resolución de $0.05^{\circ}$ (Song et al., 2018).

7.9 Freshwater Ecoregions of the World (https://www.feow.org/). Incluye una nueva regionalización de la biodiversidad de cuerpos de agua dulce, representada en un mapa. Se basa en las distribuciones y composición de especies de agua dulce (peces y en algunos casos se incluyen invertebrados y peces de agua salobre) e incorpora, además, los principales patrones ecológicos y biogeográficos (Abell et al., 2008).

7.10 Harmonized Global Land Use for Years 1500-2100, v1 (https://daac. ornl.gov/VEGETATION/guides/Land_Use_Harmonization. V1.html). Incluye información que representa el uso de suelo y cobertura vegetal para el periodo $1500-2100$ a escala global y con una resolución espacial de $0.5^{\circ}$. Los datos de cobertura de suelo para los años 1500-2005 se basan en HYDE 3.1 y las proyecciones a futuro para el periodo 2006-2100 se basan en cuatro escenarios del Modelo de Evaluación Integrada (IAM) (Chini, 2014).

7.11 Global Land Cover Facility (GLCF) (https://geog.umd.edu/ feature/global-land-cover-facility-\%28glcf\%29). Es un proyecto de investigación desarrollado por el Departamento de Ciencias Geográficas de la Universidad de Maryland. Dentro de la información que se puede encontrar son mapas históricos de la cubierta forestal y de cambios de la cubierta forestal a nivel global y local (Kim et al., 2014; Sexton et al., 2013, 2015; Song et al., 2015).

7.12 Temporally inter-comparable maps of terrestrial wilderness and the Last of the Wild (https:/datadryad.org/stash/dataset/doi:10.5061/ dryad.124fp). Es una serie de mapas actualizados de las áreas silvestres terrestres a nivel mundial que son esenciales para monitorear los cambios en su extensión desde 1993 al 2009 (Allan et al., 2017).

7.13 Terrestrial Ecoregions of the World (https://www.worldwildlife. org/publications/terrestrial-ecoregions-of-the-world). Incluye una regionalización biogeográfica de la biodiversidad terrestre. Las unidades biogeográficas son ecorregiones que reflejan la distribución de una amplia gama de fauna y flora; existen 867 unidades clasificadas en 14 biomas diferentes (Olson et al., 2001).

7.14 SILVIS Lab, Spatial Analysis for Conservation and Sustainability (http://silvis.forest.wisc.edu/). Contiene información sobre el uso de suelo y el efecto que este tiene en la conservación de la biodiversidad (particularmente en aves). Dentro de la información disponible se encuentra: a) Wildland-Urban interface (WUI) Change 1990-2010 que es una serie de mapas de Estados Unidos que ilustran la ubicación de WUI en 1990, 2000 y 2010 para apoyar la consulta sobre el efecto del crecimiento poblacional en el ambiente; cuenta con dos tipos de WUIs, las primeras son las WUI de entremezcla, que son áreas en las que las viviendas y la vegetación se unen, y las segundas son las WUI de interconexión, las cuales son áreas en las que las viviendas se localizan en la vecindad de vegetación silvestre (Stewart et al., 2007; Radeloff et al., 2005, 2018); b) Block level housing density change 1990-2010 mapas sobre el crecimiento en la densidad de viviendas en Estados Unidos entre 1990 y 2010; c) Dynamic Habitat Indices (DHI) contiene información sobre los índices de hábitat a nivel mundial con una resolución de $1 \mathrm{~km}$ calculados a partir de la información de vegetación extraída de MODIS con una temporalidad que va del 2003 hasta el 2014 (Hobi et al., 2017; Radeloff et al., 2019); d) DHls Clusters son una serie de datos en los que se agrupan el DHI acumulado anual, el mínimo y la variación de la fracción de radiación fotosintéticamente activa (fPAR por sus siglas en inglés), y la latitud (Coops et al., 2018); e) Global snow and subnivium trends datos sobre los patrones globales de la duración del suelo cubierto de nieve y la duración del suelo congelado sin nieve con diferentes resoluciones y temporalidades (Zhu et al., 2017, 2019); f) Carpathians historical land use serie de datos históricos sobre el uso de suelo para la Región Cárpatos en Europa oriental (Munteanu et al., 2015; Kaim et al., 2016); g) Impervious/tree cover change around Chicago información de la dinámica espacio-temporal de algunos accipítridos en Chicago, Estados Unidos (McCabe et al., 2018); h) Projections of future U.S. land cover in $\mathbf{2 0 5 0}$ (NSF) modelos de cambio de uso de suelo para Estados Unidos al 2050 (Radeloff et al., 2012; Lawler et al., 2014); i) Climate averages and extremes data tres escenarios climáticos para Estados Unidos de 1980-2014, 1949-2010 y 1950-2100; j) Habitat heterogeneity texture metrics datos de textura y verdor para Estados Unidos y la mitad de México; k) Land-cover change maps of the Caucasus mapas de la cobertura de suelo y cambios de uso de suelo para el Cáucaso.

7.15 World Database Protected Areas (https://www.iucn.org/theme/ protected-areas/our-work/world-database-protected-areas). Incluye las áreas protegidas terrestres y marinas a nivel mundial. Se trata de un proyecto conjunto entre el Programa de Naciones Unidas para el Medio Ambiente y la Unión Internacional para la Conservación de la Naturaleza manejado por el UNEP Conservation Monitoring Centre (UNEP-WCMC) (IUCN, 2020). 


\section{IMPACTO ANTROPOGÉNICO}

8.1 Cumulative Human Impacts to California Current Marine Ecosystems (https://knb.ecoinformatics.org/view/doi:10.5063/F11Z42N8). Incluye un mapa que representa los impactos acumulativos para 19 ecosistemas marinos presentes en la corriente de California utilizando datos de 25 actividades humanas (dividido en cuatro conjuntos: cambio climático, fuentes de estrés de origen terrestre, tipo de pesca y otras actividades comerciales relacionadas con el océano) (Halpern et al., 2009).

8.2 Global Administrative Areas (GADM) (https://gadm.org/index. html). Incluye mapas y datos espaciales para todos los países y sus subdivisiones administrativas (GADM, 2020).

8.3 Global Map of Human Impacts to Marine Ecosystems (https:// www.nceas.ucsb.edu/globalmarine). Contiene información sobre el impacto humano en los océanos para 2008 (18 variables) y 2013 (19 variables). Estas incluyen información sobre pesquerías, componentes inorgánicos, contaminación oceánica, fertilizantes, actividad mercantes (Halpern et al., 2008, 2015).

8.4 Global Road Maps (https://www.global-roadmap.org/ global-road-maps/). Serie de mapas para la priorización en la construcción de carreteras a partir de una zonificación que minimice el impacto en el ambiente y al mismo tiempo beneficiar el desarrollo humano. Muestran las áreas en las que se debería de evitar la construcción de carreteras, así como también áreas en las que la construcción de carreteras podrían promover el desarrollo agrícola con costos ambientales moderados y áreas en donde la construcción de carreteras beneficiaría la agricultura, aunque con un gran impacto en el ambiente (Laurance \& Balmford, 2013; Laurance et al., 2014).

8.5 Global Terrestrial Human Footprint maps for 1993 and 2009 (https:// datadryad.org/stash/dataset/doi:10.5061/dryad.05295). Incluye ocho variables (entornos construidos, densidad de población, infraestructura eléctrica, tierras de cultivo, tierras de pastoreo, carreteras, ferrocarriles y vías navegables) que miden las presiones humanas directas e indirectas sobre el ambiente. Los datos están disponibles para los años 1993 y 2009, con una escala global y una resolución temporal de $1 \mathrm{~km}^{2}$ (Venter et al., 2016a, b).

8.6 Global Urban Sprawling Map (https://urbanclimate.tse.ens. titech.ac.jp/2018/10/15/urban-sprawling-map/). Incluye un modelo que proyecta el crecimiento urbano a escala global para el periodo 2020-2050, con una resolución aproximada de $1 \mathrm{~km}^{2}$. Se basa en el modelo de crecimiento urbano SLEUTH y la base de datos de la distribución poblacional de LandScan y simula el crecimiento urbano que abarcan las regiones urbanas (Zhou et al., 2019).

8.7 Gridded global datasets for Gross Domestic Product and Human Development Index over 1990-2015 (https://datadryad.org/stash/ dataset/doi:10.5061/dryad.dkijo). Datos anuales del Producto Interno Bruto (PIB per cápita y PIB total) y el Índice de Desarrollo Humano (IDH). Los datos se encuentran disponibles para el periodo de 1990-2015 a escala global y con una resolución de 30" (Kummu et al., 2020).

8.8 Gridded Population of the World (GPW) v4 (https://sedac.ciesin. columbia.edu/data/collection/gpw-V4/sets/browse) Esta colección modela la distribución de la población humana a escala global y con una resolución espacial de 30". Este conjunto de datos proviene de los resultados obtenidos a partir de los censos poblacionales y de vivienda de 2010 (2005 y 2014); estos datos se fueron utilizados para producir estimaciones poblacionales para los años 2000, 2005, 2010, 2015 y 2020 (CIESIN, 2018a). Dentro de esta colección se encuentran disponibles los siguientes datos: a) Administrative Unit Center Points with Population Estimates, v4.11 datos sobre estimaciones y densidades poblacionales para los años 2000, 2005, 2010, 2015 y 2020, además, se incluyen caracterísiticas demográficas (edad y sexo) para el año 2010, por unidad administrativa (CIESIN, 2018b); b) Basic Demographic Characterics, V4.11 (2010) incluye estimaciones de población humana por edad y sexo, como recuentos (número de personas por pixel) y densidades (número de personas por $\mathrm{km}^{2}$ ), basadas en los registros de censos nacionales y registros de población para el año 2010, con una resolución de 30", 2.5', 15' y 30' y de $1^{\circ}$ (CIESIN, 2018c); c) Data Ouality Indicators, v4.11 (2010) provee el contexto para las coberturas de conteo y densidad de población, debido a que contiene información espacial específica directamente relacionada con las causas de la distribución de la población humana (CIESIN, 2018d); d) Population Count, v4.1 (2000, 2005, 2010, $\mathbf{2 0 1 5}, \mathbf{2 0 2 0}$ ) contiene información sobre estimaciones de población humana (número de personas por pixel), basadas en los registros de censos nacionales y registros de población para los años 2000 , 2005, 2010, 2015 y 2020 (CIESIN, 2018e); e) Land and Water Area, v4.11 (2010) son dos mapas que representan la superficie de tierra (área terrestre, excluyendo hielo y cuerpos de agua permanentes) y agua (incluyendo hielo y cuerpos de agua permanentes) en $\mathrm{km}^{2}$ por pixel (CIESIN, 2018f); f) National Identifier Grid, v 4.11 (2010) es un mapa de estados-naciones para su uso en la agregación de datos de población. Este conjunto de datos se produjo a partir de registros de censos nacionales (CIESIN, 2018g); g) UN WPP - Adjusted Population Count, v 4.11 (2000, 2005, 2010, 2015, 2020) datos sobre estimaciones de población humana (número de 
personas por pixel) de acuerdo con los conteos realizados por los censos nacionales y los registros de población con respecto a la distribución relativa espacial pero ajustada, para que coincida con la revisión de 2015 de los totales de las Naciones Unidas para las Perspectivas de Población Mundial para los años 2000, 2005, 2010, 2015 y 2020 (CIESIN, 2018h); h) UN WPP - Adjusted Population Density, v $4.11(2000,2005,2010,2015,2020)$ incluye estimaciones de densidad poblacional humana (número de personas por $\mathrm{km}^{2}$ ) basados en conteos realizados por los censos poblacionales y registros poblacionales con respecto a la distribución espacial relativa pero ajustada para que coincida con la revisión de 2015 de los totales de las Naciones Unidas para las Perspectivas de Población Mundial para los años 2000, 2005, 2010, 2015 y 2020 (CIESIN, 2018i); i) Population Density, v4.11 (2000, 2005, 2010, 2015, 2020) incluye estimaciones de la densidad de población humana (número de personas por $\mathrm{km}^{2}$ ) basados en conteos realizados por los censos poblacionales y registros poblacionales para los años 2000, 2005, 2010, 2015 y 2020 (CIESIN, 2018j).

8.9 Mapa de Afectación Humana (2009) (https:// drive.google.com/drive/folders/ i E u Y Yo8v7t8AYouIgc4vqlNqXOaLErmp?usp=sharing). Archivo en formato raster que integra una representación cartográfica de la afectación humana sobre el territorio terrestre mexicano. Incluye metadatos y dos proyecciones (Bezaury-Creel \& OchoaOchoa, 2009).

8.10 PEST-CHEMGRIDS (https://www.nature.com/articles/s41597019-0169-4\#Abs1). Contiene datos de los 20 ingredientes activos de pesticidas más utilizados en seis cultivos y cuatro conjuntos de cultivos. Los datos están disponibles a una escala global y una resolución espacial de 5' proyectada para los años 2015-2025. Los datos se derivaron de las bases de datos de plaguicidas USGS/ PNSP y FAOSTAT, junto con otros inventarios públicos (incluidos datos de propiedades físicas del suelo, variables hidroclimáticas, cantidades agrícolas e índices socioeconómicos) (Maggi et al., 2019).

8.11 Recent pace of change in human impact on the world's ocean (https://knb.ecoinformatics.org/view/doi:10.5063/F12B8WBS). Incluye el cálculo y mapas del impacto acumulativo de 14 factores estresantes relacionados con la actividad humana (incluyendo el cambio climático, la pesca, las presiones terrestres y otras actividades comerciales) y su impacto en 21 ecosistemas marinos para el periodo 2003-2013. Los datos están disponibles a escala global y con una resolución espacial de $1 \mathrm{~km}^{2}$ (Halpern et al., 2019).

\section{MULTIPLES}

9.1 Bio-ORACLE (http://www.bio-oracle.org/). Contiene información de 23 variables geofísicas, bióticas y climáticas que incluyen temperatura, salinidad, nutrientes, clorofila, capa de hielo, velocidad de la corriente, fitoplancton, productividad primaria, hierro y penetración de la luz. Las variables presentan una resolución de 5' con una escala a nivel global. Tiene capas marinas a nivel superficial, en diferentes capas bentónicas, condiciones actuales $y / o$ en escenarios futuros. Presenta un paquete llamado "sdmpredictors" con funciones en el entorno de $\mathrm{R}$ software para facilitar la extracción y gestión de las variables (Tyberghein et al., 2012; Assis et al., 2017).

9.2 CGIAR Consortium of Spatial Information (CGIAR-CSI). Incluye información de:a)Global Aridity Index and Potential Evapotranspiration Climate Database v2 (https://cgiarcsi.community/2019/01/24/ global-aridity-index-and-potential-evapotranspirationclimate-database-v2/) Variables climáticas relacionadas con la evapotranspiración y la aridez (1970-2000). Está basado en implementación de la ecuación de evapotranspiración de referencia de Penman-Montieth, a una escala casi global (excluyendo la Antártida) y una resolución espacial de 30" (Zomer et al., 2006, 2008; Trabucco \& Zomer, 2019a); b) Global High-Resolution Soil-Water Balance (https://cgiarcsi.community/ data/global-high-resolution-soil-water-balance/), corresponde a datos hidrológicos que describen la evapotranspiración y el déficit hídrico del suelo, con una escala global y una resolución espacial de 30 segundos de arco $(\sim 1 \mathrm{~km})$. Los datos se derivaron de WorldClim y Global-PET (Trabucco \& Zomer, 2019b).

9.3 Instituto Geográfico Agustín Codazzi (Colombia) (https:// geoportal.igac.gov.co/contenido/datos-abiertos-igac). Contiene una serie de mapas oficiales de Colombia, además de elaborar el catastro nacional de la propiedad inmueble, el inventario de las características del suelo, entre otros. El conjunto de datos se encuentra disponibles con las siguientes escalas: 1:500 000, 1:100 000 y $1: 25000$ (IGAC, 2020).

9.4 Instituto Nacional de Estadística y Geografía (México) (https://www. inegi.org.mx/). Incluye información geográfica y estadística a nivel estatal y nacional. Existe información cartográfica sobre topografía, hidrografía, hidrología, geología, fisiografía, climatología, edafología, vías de comunicación, uso de suelo y vegetación, uso potencial de suelo y humedales potenciales. La información cartográfica puede encontrarse en las escalas 1:1 $000000,1: 250000,1: 50000$ y 1:20 000 (INEGI, 2020). 
9.5 Dynamic Ecological Information Management System-Site and dataset registry (DEIMS-SDR) (https://deims.org/). Incluye el registro de sitios de investigación y monitoreo ambiental que permite descubrir sitios de investigación de ecosistemas a largo plazo en todo el mundo, gracias a los datos obtenidos in situ y a las personas o redes asociados con ellos. Este sistema proporciona una gran cantidad de información (incluyendo la ubicación de cada sitio, ecosistemas, temas de investigación, etc.) (Wohner et al., 2019).

9.6 EarthEnv (http://www.earthenv.org/). Es un proyecto desarrollado por expertos en biodiversidad y en teledetección para la obtención de capas casi globales con una resolución de 1 $\mathrm{km}^{2}$ para realizar el monitoreo y modelación de la biodiversidad, ecosistemas y clima. Dentro de este se encuentra: a) Near-global environmental information for freshwater ecosystems in $1 \mathrm{~km}$ resolution (http://www.earthenv.org/streams), el cual incluye variables ambientales (entre ellas topográficas: elevación y pendiente) relacionadas a cuerpos de agua dulce, con una escala casi global $\left(60^{\circ} \mathrm{N}\right.$ a $5^{\circ} \mathrm{S}$ y $145^{\circ} \mathrm{O}$ a $\left.180^{\circ} \mathrm{E}\right)$ y una resolución espacial de $1 \mathrm{~km}$ (Domisch et al., 2015); b) Global 1, 5, 10, 100 km Topography (http:// www.earthenv.org/topography) que contiene información a nivel global de variables con diferentes resoluciones $(1,5,10,50$ y 10 $\mathrm{km}$ ) como elevación, pendiente, aspecto, este, norte, rugosidad, índice de rugosidad del terreno, índice de posición topográfica, medida de rugosidad del vector, curvatura del perfil/tangencial, derivada parcial de primer/segundo orden y 10 clases de formas geomorfológicas (Amatulli et al., 2018); c) EarthEnv-DEM90 digital elevation model (http://www.earthenv.org/DEM) es un modelo digital de elevación con resolución de $90 \mathrm{~m}$ (Robinson et al., 2014); d) Global 1 km- Cloud Cover (http://www.earthenv.org/cloud) con variables que describen la dinámica mensual de nubes durante un periodo de 15 años (2000-2014) a partir de dos observaciones diarias, en una escala casi global y una resolución espacial de 1 $\mathrm{km}^{2}$ (Wilson \& Jetz, 2016); e) Global Habitat Heterogeneity (http:// www.earthenv.org/texture), incluye 14 variables que cuantifican la heterogeneidad del hábitat: seis de primer orden, las cuales describen la distribución de la frecuencia de los valores de Imágenes del Índice de Vegetación Mejorado (EVI por sus siglas en inglés) y cuantifican la variabilidad en EVI dentro de un área; y ocho de segundo orden, las cuales consisten en estadísticos que describen las probabilidades de ocurrencia de diferentes combinaciones de EVI entre pares de píxeles dentro de un área. Este conjunto de datos se basa en las características texturales de EVI obtenidas de MODIS. Estos datos se encuentran disponibles a escala global y una resolución espacial de 30 ", 2.5 y 12.5 minutos de arco (Tuanmu \& Jetz, 2015); f) Global 1 km Consensus Land Cover (http://www.earthenv.org/landcover), la cual incluye dos versiones que contienen 12 capas de datos que describen la cobertura del suelo. Estos datos se derivaron de la teledetección y se encuentran disponibles a una escala casi global (excepto la Antártida), con una resolución espacial de $1 \mathrm{~km}^{2}$ (Tuanmu \& Jetz, 2014).

9.7 Earth Explorer (EE) (https://earthexplorer.usgs.gov/). Interfaz desarrollada por el Servicio Geológico de Estados Unidos (USGS) que permite al usuario buscar y/o descubrir inventarios información derivada de satélites, aeronaves y otros sensores remotos (USGS, 2020).

9.8 ENVIREM v1.0 (https://envirem.github.io/). Conjunto de datos con información climática y topográfica. La información climática corresponde a datos para el presente (1960-1990) y reconstrucciones paleoclimáticas (Holoceno medio y UMG), se tienen varias regiones geográficas que cubren (excepto la Antártida) y una resolución de 30", 2.5', 5' y 10' (Title \& Bemmels, 2018).

9.9 MARSPEC: Ocean Climate database (http://www.marspec.org/). Incluye 17 variables bioclimáticas a nivel marino (derivadas de temperatura, concentración de nutrientes y topografía), con una escala global y una resolución espacial de $30^{\prime \prime}, 2.5^{\prime}, 5^{\prime}$ y $10^{\prime}$. Estas capas se derivaron del World Ocean Atlas de NOAA y de las imágenes satelitales MODIS. Además, se incluyen proyecciones para el pasado (Holoceno medio y UMG), con una escala global y una resolución espacial de 5' (Sbrocco \& Barber, 2013; Sbrocco, 2014).

9.10 Portal de Geoinformación 2020. Sistema Nacional de Infomación sobre Biodiversidad (SNIB-CONABIO) (http://www.conabio.gob.mx/ informacion/gis/). Incluye información sobre grupos biológicos (aves, mamíferos, anfibios, reptiles, peces, invertebrados, hongos, plantas, protoctistas y bacterias), información de biodiversidad, topografía, hidrología, edafología, geología, climatología, vegetación y uso de suelo, división política, población, infraestructura, regionalización, productos satelitales, riesgo y producción (CONABIO, 2020).

9.11 World Ocean Atlas 2018 (W0A18) (https://www.nodc.noaa. gov/OC5/woa18/). Incluye información de 102 profundidades estándar para el océano, con una escala global y una resolución de $0.25^{\circ}$ y $^{\circ}$ sobre temperatura (Locarnini et al., 2018), salinidad (Zweng et al., 2018), distribución climatológica de oxígeno disuelto, utilización aparente de oxígeno y saturación de oxígeno disuelto (Garcia et al., 2018a), distribución de nutrientes (fosfato, nitrato, nitrato+nitrito y silicato) (Garcia et al., 2018b). 


\section{LITERATURA CITADA}

Abatzoglou, J.T., S.Z. Dobrowski, S.A. Parks \& K.C. Hegewisch. 2018. Terraclimate, a high-resolution global dataset of monthly climate and climatic water balance from 1958-2015. Scientific Data 5:170191.

Abell, R., M.L. Thieme, C. Revenga, M. Bryer, M. Kottelat, N. Bogutskaya, B. Coad, N. Mandrack, S. Contreras, W. Bussing, M.L.J. Stiassny, P. Skelton, G.R. Allen, P. Unmack, A. Naseka, R. Ng, N. Sindorf, J. Robertson, E. Armijo, J.V. Higgins, T.J. Heibel, E. Wikramanayake, D. Olson, H.L. López, R.E. Reis, J.G. Lundberg, M.H. Sabaj \& P. Petry. 2008. Freshwater Ecoregions of the World: A New Map of Biogeographic Units for Freshwater Biodiversity Conservation. BioScience 58:403-414.

Allan, J.R., O. Venter \& E.M. Watson. 2017. Temporally intercomparable maps of terrestrial wilderness and the Last of the Wild. Scientific Data 4:170187.

Allen, G.H. \& T.M. Pavelsky. 2018. Global extent of rivers and streams. Science 361:585-588.

Amante, C. \& B.W. Eakins. 2009. ETOPO1 1 Arc-Minute Global Relief Model: Procedures, Data Sources and Analysis. NOAA Technical Memorandum NESDIS NGDC-24. National Geophysical Data Center, NOAA.

Amatulli, G., S. Domisch, M.N. Tuanmu, B. Parmentier, A. Ranipeta, J. Malczyk \& W. Jetz. 2018. A suite of global, crossscale topographic variables for environmental and biodiversity modeling. Scientific Data 5:180040.

Amatulli, G., D. McInerney, T. Sethi, P. Strobl \& S. Domisch. 2019. Data Descriptor: Geomorphogom - Global high-resolution geomorphometry layers: empirical evaluation and accuracy assessment. PeerJ Preprints 7:e27595v1.

Assis, J., L. Tyberghein, S. Bosh, H. Verbruggen, E.A. Serrão \& O. de Clerck, (2017). Bio-ORACLE v2.o: Extending marine data layers for bioclimatic modelling. Global Ecology and Biogeography $27: 277-284$

Batjes, N.H., E. Ribeiro \& A.J.M. van Oostrum. 2020. Standardised soil profile data to support global mapping and modelling (WoSIS snapshot 2019). Earth System Science Data 12:299-320.
Batjes, N.H., E. Ribeiro, A. van Oostrum, J. Leenaars, T. Hengl \& J. Mendes de Jesus. 2017. WoSIS: providing standardised soil profile data for the world. Earth System Science Data 9:1-14.

Beck, H.E., N.E. Zimmermann, T.R. McVicar, N. Vergopolan, A. Berg \& E.F. Wood. 2018. Present and future Köppen-Geiger climate classification maps at $1 \mathrm{~km}$ resolution. Scientific Data 5:180214.

Bezaury-Creel, J.E. \& L.M. Ochoa-Ochoa. 2009. Base de Datos Geográfica de la Afectación Humana sobre el Territorio Mexicano - Versión 1.0, 07/2009. 2 capas formato ráster + 1 capa Goggle Earth KMZ + 1 Archivo de Metadatos Word.

Bezaury-Creel J.E., L.M. Ochoa-Ochoa \& J.F. Torres-Origel. 2012. Base de Datos Geográfica de las Reservas de Conservación Privadas y Comunitarias en México - Versión 2.0 The Nature Conservancy. 2 Capas ArcGIS 9.2 + 1 Capa Google Earth KMZ + 1 Archivo de Metadatos en texto.

Bittig H.C., T. Steinhoff, H. Claustre, B. Fiedler, N.L. Williams, R. Sauzède, A. Körtzinger \& J.P. Gattuso, 2018. An Alternative to Static Climatologies: Robust Estimation of Open Ocean $\mathrm{CO}_{2}$ Variables and Nutrient Concentrations From T, S, and $\mathrm{O}_{2}$ Data Using Bayesian Neural Networks. Frontiers in Marine Science $5: 328$

Bontemps, S., M. Boettcher, C. Brockman, G. Kirches, C. Lamarche, J. Radoux, M. Santoro, E. Van Bogaert, U. Wegmüller, M. Herold, F. Ramoino, O. Arino \& P. Defourny. 2015. Multi-year global land cover mapping at $300 \mathrm{~m}$ and characterization for climate modelling: achievements of the land cover component of the ESA climate change initiative. Pp. 323-328. En International Archives of the Photogrammetry, Remote Sensing \& Spatial Information Sciences. Berlin, Alemania.

Bontemps, S., P. Defourny, J. Radoux, E. Van Bogaert, C. Lamarche, F. Achard, P. Mayaux, M. Boettcher, C. Brockmann, G. Kirches, M. Zülkhe, V. Kalogirou, F.M. Seifert \& O. Arino. 2013. Consistent global land cover maps for climate modelling communities: current achievements of the ESA' Land Cover CCI. Pp. 9-13. En Proceedings of the ESA Living Planet Symposium, Edimburgh.

Brown, J.L., D.J. Hill, A.M. Dolan, A.C. Carnaval \& A.M. Haywood. 2018. PaleoClim, high spatial resolution paleoclimate surfaces for global land areas. Scientific Data 5:18025.

Chini, L.P., G.C. Hurtt \& S. Frolking. 2014. Harmonized Global Land Use for Years 1500-2100, V1. ORNL DAAC, Oak Ridge, Tennessee, 
USA. http://dx.doi.org/10.3334/ORNLDAAC/1248 [Consultado en Abril 2020].

CIESIN (Center for International Earth Science Information Network-Columbia University). 2018a. Documentation for the Gridded Population of the World, Version 4 (GPWv4), Revision 11 Data Sets. Palisades NY: NASA Socioeconomic Data and Applications Center (SEDAC). https://doi.org/10.7927/H45Q4T5F [Consultado en Abril 2020].

CIESIN (Center for International Earth Science Information Network-Columbia University). 2018b. Gridded Population of the World, Version 4 (GPWv4): Administrative Unit Center Points with Population Estimates, Revision 11. Palisades, NY: NASA Socioeconomic Data and Applications Center (SEDAC). https:// doi.org/10.7927/H4BC3WMT [Consultado en Abril 2020].

CIESIN (Center for International Earth Science Information Network-Columbia University). 2018c. Gridded Population of the World, Version 4 (GPWv4): Basic Demographic Characteristics, Revision 11. Palisades, NY: NASA Socioeconomic Data and Applications Center (SEDAC). https://doi.org/10.7927/ H46M34XX [Consultado en Abril 2020].

CIESIN (Center for International Earth Science Information Network-Columbia University). 2018d. Gridded Population of the World, Version 4 (GPWv4): Data Quality Indicators, Revision 11. Palisades, NY: NASA Socioeconomic Data and Applications Center (SEDAC). https://doi.org/10.7927/H42Z13KG [Consultado en Abril 2020].

CIESIN (Center for International Earth Science Information Network-Columbia University). 2018e. Gridded Population of the World, Version 4 (GPWv4): Population Count, Revision 11. Palisades, NY: NASA Socioeconomic Data and Applications Center (SEDAC). https://doi.org/10.7927/H4JW8BX5 [Consultado en Abril 2020].

CIESIN (Center for International Earth Science Information Network-Columbia University). 2018f. Gridded Population of the World, Version 4 (GPWv4): Land and Water Area, Revision 11. Palisades, NY: NASA Socioeconomic Data and Applications Center (SEDAC). https://doi.org/10.7927/H4Z60M4Z [Consultado en Abril 2020].

CIESIN (Center for International Earth Science Information Network-Columbia University). 2018g. Gridded Population of the World, Version 4 (GPWv4): National Identifier Grid, Revision 11. Palisades, NY: NASA Socioeconomic Data and Applications
Center (SEDAC). https://doi.org/10.7927/H4TD9VDP [Consultado en Abril 2020].

CIESIN (Center for International Earth Science Information Network-Columbia University). 2018h. Gridded Population of the World, Version 4 (GPWv4): Population Count Adjusted to Match 2015 Revision of UN WPP Country Totals, Revision 11. Palisades, NY: NASA Socioeconomic Data and Applications Center (SEDAC). https://doi.org/10.7927/H4PN93PB [Consultado en Abril 2020].

CIESIN (Center for International Earth Science Information Network-Columbia University). 2018i. Gridded Population of the World, Version 4 (GPWv4): Population Density Adjusted to Match 2015 Revision UN WPP Country Totals, Revision 11. Palisades, NY: NASA Socioeconomic Data and Applications Center (SEDAC). https://doi.org/10.7927/H4F47M65 [Consultado en Abril 2020].

CIESIN (Center for International Earth Science Information Network-Columbia University). 2018j. Gridded Population of the World, Version 4 (GPWv4): Population Density, Revision 11. Palisades, NY: NASA Socioeconomic Data and Applications Center (SEDAC). https://doi.org/10.7927/H49C6VHW [Consultado en Abril 2020].

CONABIO (Comisión Nacional para el Conocimiento y uso de la Biodiversidad). 2020. Portal de Geoinformación 2020. Sistema Nacional de Información sobre Biodiversidad. http://www. conabio.gob.mx/informacion/gis/ [Consultada en Abril 2020].

CONANP (Comisión Nacional de Áreas Naturales Protegidas) 2020. http://sig.conanp.gob.mx/website/pagsig/datos_anp.htm [Consultado en Abril 2020].

Coops, N.C., S.P. Kearney, D.K. Bolton \& V.C. Radeloff. 2018 Remotely-sensed productivity clusters capture global biodiversity patterns. Scientific Reports 8:16261.

Cruz-Cárdenas, G., L. López-Mata, C.A. Ortiz-Solorio, J.L. Villaseñor, E. Ortiz, J.T. Silva \& F. Estrada-Dodoy. 2014 Interpolation of Mexican soil properties at a scale of 1:1,000,000. Geoderma 213:29-35.

Cuervo-Robayo, A.P. 2019, Marzo 22. En Facebook. https://www.facebook.com/groups/245646162181621/ permalink/2187339168012301/ [Consultado en Abril 2020].

Cuervo-Robayo, A.P., O. Téllez-Valdés, M.A. Gómez-Albores, C.S Venegas-Barrera, J. Manjarrez \& E. Martínez-Meyer. 2014. An 
update of high-resolution monthly climate surfaces for Mexico. International Journal of Climatology 34:2427-2437.

Danielson, J.J. \& D.B. Gesch. 2011. Global multi-resolution terrain elevation data 2010 (GMTED2010). U.S. Geological Survey OpenFile Report 2011-1073.

Defourny, P., S. Bontemps, C. Brockman, M. Herold, G. Kirches, C. Lamarche, V. Kalogirou, F.M. Seifert \& O. Arino. 2012. Land cover CCI. Product User Guide Version 2.

Domisch, S., G. Amatulli \& W. Jetz. 2015. Near-global freshwaterspecific environmental variables for biodiversity analyses in $1 \mathrm{~km}$ resolution. Scientific Data 2:150073.

Emetere, M.E. \& E.T. Akinlabi. 2020. Introduction to Environmental Data Analysis and Modeling. Springer, Cham, Suiza.

EMODnet Bathymetry Consortium (2018). EMODnet Digital Bathymetry (DTM 2018). EMODnet Bathymetry Consortium. https://doi.org/10.12770/18ffod48-b203-4a65-94a9-5fd8boec35f6 [Consultado en Abril 2020].

Fick, S.E. \& R.J. Hijmans. 2017. WorldClim 2: new $1 \mathrm{~km}$ spatial resolution climate surfaces for global land areas. International Journal of Climatology 37:4302-4315.

Fore, A.G, S.H. Yueh, W. Tang, B.W. Stiles \& A.K. Hayashi. 2016. Combined Active/Passive Retrievals of Ocean Vector Wind and Sea Surface Salinity With SMAP. IEEE Transactions on Geoscience and Remote Sensing 54:7396-7404.

Funk, C., P. Peterson, M. Landsfield, D. Pedreros, J. Vedin, S. Shukla, G. Husak, J. Rowald, L. Harrison, A. Hoell \& J. Michaelsen. 2015. The climate hazards infrared precipitation with stations-a new environmental record for monitoring extremes. Scientific Data 2:150066.

GADM (Global Administrative Areas). 2020. Download. Global Administrative Areas GADM version 3.6 http:/gadm.org/ country [Consultado en Abril 2020].

Garcia, H.E., K. Weathers, C.R. Paver, I. Smolyar, T.P. Boyer, R.A. Locarnini, M.M. Zweng, A.V. Mishonov, O.K. Baranova, D. Seidov \& J.R. Reagan. 2018a. World Ocean Atlas 2018, Volume 3: Dissolved Oxygen, Apparent Oxygen Utilization, and Oxygen Saturation. NOAA Atlas NESDIS 83, Silver Spring, Maryland, USA.
Garcia, H.E., K. Weathers, C.R. Paver, I. Smolyar, T.P. Boyer, R.A. Locarnini, M.M. Zweng, A.V. Mishonov, O.K. Baranova, D. Seidov \& J.R. Reagan. 2018b. World Ocean Atlas 2018, Volume 4: Dissolved Inorganic Nutrients (phosphate, nitrate and nitrate+nitrite, silicate). NOAA Atlas NESDIS 84, Silver Spring, Maryland, USA.

GEBCO Bathymetric Compilation Group 2019. 2019. The GEBCO_2019 Grid - a continuous terrain model of the global oceans and land. British Oceanographic Data Centre, National Oceanography Centre, NERC, UK.

Hadley Centre for Climate Prediction and Research. 2006. Met Office HadISST1.1-Globalsea-Ice coverageand Sea Surface Temperature (1870-2015), NCAS British Atmospheric Data Centre. http:// catalogue.ceda.ac.uk/uuid/facafa2ae494597166217a9121a62d3c [Consultado en Abril 2020].

Halpern, B.S., M. Frazier, J. Afflerbach, J.S. Lowndes, F. Micheli, C. O'Hara, C. Scarborough \& K.A. Selkoe. 2019. Recent pace of change in human impact on the world's ocean: Cumulative impacts. Knowledge Network for Biocomplexity. Scientific Reports 9:11609.

Halpern, B.S., M. Frazier, J. Potapenko, K.S. Casey, K. Koenig, C. Longo, J.S. Lowndes, R.C. Rockwood, E.R. Selig, K.A. Selkoe \& S. Walbridge. 2015. Spatial and temporal changes in cumulative human impacts on the world's ocean. Nature Communications 6:7615.

Halpern, B.S., C.V. Kappel, K.A. Selkoe, F. Michelli, C.M. Ebert, C. Kontgis, C.M. Crain, R.G. Martone, C. Shearer \& S.J. Teck. 2009. Mapping cumulative human impacts to California Current marine ecosystems. Conservation Letters 2:138-148.

Halpern, B.S., S. Walbridge, K.A. Selkoe, C.V. Kappel, F. Micheli, C. Agrosa, J.F. Bruno, K.S. Casey, C. Ebert, H.E. Fox, R. Fujita, D. Heinemann, H.S. Lenihan, E.M.P. Madin, M.T. Perry, E.R. Selig, M. Spalding, R. Steneck \& R. Watson. 2008. A Global Map of Human Impact on Marine Ecosystems. Science 319:948-952.

Hamann, A., T. Wang, D.L. Spittlehouse \& T.Q. Murdock. 2013 A comprehensive, high-resolution database of historical and projected climate surfaces for western North America. Bulletin of the American Meteorological Society 94:1307-1309.

Hansen, M.C., P.V. Potapov, R. Moore, M. Hancher, S.A.Turubanova, A. Tyukavina, D. Thau, S.V. Stehman, S.J. Goetz, T.R. Loveland, A.Kommareddy, A. Egorov, L. Chini, C.O. Justice \& J.R.G. 
Townshend. 2013. High-Resolution Global Maps of 21st-Century Forest Cover Change. Science 342:850-853.

Hao, Z., A. AghaKouchak, N. Nakhjiri \& A. Farahmand. 2014. Global Integrated Drought Monitoring and Prediction System. Scientific Data 1:140001.

Harris, I.C. \& P.D. Jones. 2019. CRU TS3.26: Climatic Research Unit (CRU) Time-Series (TS) Version 3.26 of High-Resolution Gridded Data of Month-by-month Variation in Climate (Jan. 1901Dec. 2017). Centre for Environmental Data Analysis. https:// catalogue.ceda.ac.uk/uuid/7ad889f2cc1647efba7e6a356098e4f3 [Consultado en Abril 2020].

Harris, I., P.D. Jones, T.J. Osborn \& D.H. Lister. 2014. Updated highresolution grids of monthly climatic observations-the CRU TS 3.10 Dataset. International Journal of Climatology 34:623-642.

Hengeveld, G.M., K. Gunia, M. Didion, S. Zudin, A.P.P.M. Clerkx \& M.J. Schelhaas. 2015. Global 1-degree Maps of Forest Area, Carbon Stocks, and Biomass, 1950-2010. ORNL DAAC, Oak Ridge, Tennessee, USA. http://dx.doi.org/10.3334/ORNLDAAC/1296 [Consultado en Abril 2020].

Hengl, T., J. Mendes de Jesus, G.B.M. Heuvelink, M. Ruiperez Gonzalez, M. Kilibarda, A. Blagotić, W. Shangguan, M.N. Wright, X. Geng, B. Bauer-Marschallinger, M.A. Guevara, R. Vargas, R.A. MacMillan, N.H. Batjes, J.G.B. Leenaars, E. Ribeiro, I. Wheeler, S. Mantel \& B. Kempen. 2017. SoilGrids250m: Global gridded soil information based on machine learning, PLoS ONE 12:e0169748.

Hijmans, R.J., S.E. Cameron, J.L. Parra, P.G. Jones \& A. Jarvis. 2005. Very high resolution interpolated climate surfaces for global land areas. International Journal of Climatology 25:1965-1978.

Hobi, M.L., M. Dubinin, C.H. Graham, N.C. Coops, M.K. Clayton, A.M. Pidgeon \& V.C. Radeloff. 2017. A comparison of Dynamic Habitat Indices derived from MODIS products as predictors of avian species richness. Remote Sensing of Environment 195:142152.

IGAC (Instituto Geográfico Agustin Codazzi). 2020. https:// geoportal.igac.gov.co/contenido/datos-abiertos-cartografia-ygeografia [Consultado en Abril 2020].

INEGI (Instituto Nacional de Estadística y Geografía). https://www. inegi.org.mx/default.html [Consultado en Abril 2020].
IUCN (International Union of Conservation of Nature). 2020. World Database Protected Areas (https://www.iucn.org/theme/ protected-areas/our-work/world-database-protected-areas). [Consultado en Abril 2020].

Jarvis, A., H.I. Reuter, A. Nelson \& E. Guevara. 2008. Hole-filled SRTM for the globe Version 4, available from the CGIAR-CSI SRTM 9om Database. http://srtm.csi.cgiar.org [Consultado en Abril 2020].

JPL. 2020. JPL SMAP Level 3 CAP Sea Surface Salinity Standard Mapped Image 8-Day Running Mean V4.3 Validated Dataset. Ver. 4.3. PO.DAAC, California, USA. https://doi.org/10.5067/ SMP43-3TPCS [Consultado en Abril 2020].

JPL MUR MEaSUREs Project. 2015. GHRSST Level 4 MUR Global Foundation Sea Surface Temperature Analysis (v4.1). Ver. 4.1. PO.DAAC, California, USA. https://doi.org/10.5067/GHGMR4FJ04 [Consultado en Abril 2020].

Kaim, D., J. Kozaka, N. Koleckaa, E. Ziółkowskaa, K. Ostafina, K. Ostapowicza, U. Gimmi, C. Munteanu \& V.C. Radeloff. 2016. Broad scale forest cover reconstruction from historical topographic maps. Applied Geography 67:39-48.

Karger, D.N., O. Conrad, J. Böhner, T. Kawohl, H. Kreft, R.W. Soria-Auza, N.E. Zimmermann, H.P. Linder \& M. Kessler. 2017. Climatologies at high resolution for the earth's land surface areas. Scientific Data 4:170122.

Kearney, M.R., P.I. Andrew \& P.P. Warren. 2014. microclim: Global estimates of hourly microclimate based on long-term monthly climate averages. Scientific Data 1:140006.

Kim, D.H., J.O. Sexton, P. Noojipady, C. Huang, A. Anand, S. Channan, M. Feng \& J.R. Townshend. 2014. Global, Landsatbased forest-cover change from 1990 to 2000 . Remote Sensing of Environment 155:178-193.

Kriticos, D.J., B.L. Webber, A. Leriche, N. Ota, I. Macadam, J. Bathols \& J.K. Scott. 2012. CliMond: global high-resolution historical and future scenario climate surfaces for bioclimatic modelling. Methods in Ecology and Evolution 3:53-64.

Kummu, M., M. Taka \& J.H.A. Guillaume. 2020. Data from: Gridded global datasets for Gross Domestic Product and Human Development Index over 1990-2015, v2, Dryad Dataset, https:// doi.org/10.5061/dryad.dk1jo [Consultado en Abril 2020]. 
Laurance, W.F. \& A. Balmford. 2013. A global map for road building. Nature 495:308-309.

Laurance, W.F., G.R. Clements, S. O'connell, N.D. Mueller, M. Goosem, O. Venter, D.P. Edwards, B. Phalan, A. Balmford, R. Van Der Ree \& I.B. Arrea. 2014. A global strategy for road building. Nature 513:229-232.

Lawler, J., D. Lewis, E. Nelson, A.J. Plantinga, S. Polasky, J. Withey, D. Helmers, S. Martinuzzi \& V.C. Radeloff. 2014. Projected land-use change impacts on ecosystem services in the U.S. Proceedings of the National Academy of Science 111:7492-7497.

Lehner, B., K. Verdin \& A. Jarvis. 2008. New global hydrography derived from spaceborne elevation data. Eos, Transactions, American Geophysical Union 89:93-94.

Li, W., P. Ciais, N. MacBean, S. Peng, P. Defourny \& S. Bontemps. 2016. Major forest changes and land cover transitions based on plant functional types derived from the ESA CCI Land Cover product. International Journal of Applied Earth Observation and Geoinformation 47:30-39.

Li, W., N. MacBean, P. Ciais, P. Defourny, C. Lamarche, S. Bontemps, R.S. Houghton \& S. Peng. 2018. Gross and net land cover changes in the main plant functional types derived from the annual ESA CCI land cover maps (1992-2015). Earth System Science Data 10:219-234.

Lima-Ribeiro, M.S., S. Varela, J. González-Hernández, G. de Oliveira, J.A.F. Diniz-Filho \& L.C. Terribile. 2015. ecoClimate: a database of climate data from multiple models for past, present, and future for Macroecologists and Biogeographers. Biodiversity Informatics 10:1-21.

Lima-Ribeiro, M.S., S. Varela, J. González-Hernández, G. de Oliveira, J.A.F. Diniz-Filho, A.T. Peterson \& L.C. Terribile. 2020. The ecoClimate Database. http://ecoclimate.org, [Consultado en Abril 2020].

Locarnini, R.A., A.V. Mishonov, O.K. Baranova, T.P. Boyer, M.M. Zweng, H.E. Garcia, J.R. Reagan, D. Seidov, K. Weathers, C.R. Paver \& I. Smolyar. 2018. World Ocean Atlas 2018, Volume 1: Temperature. NOAA Atlas NESDIS 81, Silver Spring, Maryland, USA.

Lorenz, D.J., D. Nieto-Lugilde, J.L. Blois, M.C. Fitzpatrick \& J.W. Williams. 2016a. Data from: Downscaled and debiased climate simulations for North America from 21,000 years ago to $2100 \mathrm{AD}$. Scientific Data 3:160048.

Lorenz, D.J., D. Nieto-Lugilde, J.L. Blois, M.C. Fitzpatrick \& J.W. Williams. 2016b. Data from: Downscaled and debiased climate simulations for North America from 21,000 years ago to 2100AD, v2, Dryad Dataset. https://doi.org/10.5061/dryad.1597g [Consultado en Abril 2020].

Maggi, F., F.H.M. Tang, D. la Cecilia \& A. McBratney. 2019. PESTCHEMGRIDS, global gridded maps of the top 20 crop-specific pesticide application rates from 2015 to 2025 . Scientific Data 6:170.

McCabe, J.D., H. Yin, J. Cruz, V. Radeloff, A. Pidgeon, D.N. Bonter \& B. Zuckerbeg. 2018. Prey abundance and urbanization influence the establishment of avian predators in a metropolitan landscape. Proceedings of the Royal Society B 285:20182120.

Merchant, C.J., O. Embury, C.E. Bulgin, T. Block, G.K. Corlett, E Fiedler, S.A. Good, J. Mittaz, N.A. Rayner, D. Berry, S. Eastwood, M. Taylor, Y. Tsushima, A. Waterfall, R. Wilson \& C. Donlon. 2019a. Metadata record for: Satellite-based time-series of seasurface temperature since 1981 for climate applications. https:// doi.org/10.6084/m9.figshare.9939638.v2 [Consultado en Abril 2020].

Merchant, C.J., O. Embury, C.E. Bulgin, T. Block, G.K. Corlett, E. Fiedler, S.A. Good, J. Mittaz, N.A. Rayner, D. Berry, S. Eastwood, M. Taylor, Y. Tsushima, A. Waterfall, R. Wilson \& C. Donlon. 2019b. Satellite-based time-series of sea-surface temperature since 1981 for climate applications. Scientific Data 6:223.

Munteanu, C., T. Kuemmerle,N.S. Keuler, D. Müller, P. Balázs, M. Dobosz, P. Griffiths, L. Halada, D. Kaim, G. Király, E. KonkolyGyuró, J. Kozak, J. Lieskovsky, K. Ostafin, K. Ostapowicz, O. Sandra \& V.C. Radeloff. 2015. Legacies of 19th century land use shape contemporary forest cover. Global Environmental Change 34:83-94.

NASA-GSFC-OBPG (National Aeronautics and Space Administration Goddard Space Flight Center, Ocean Biology Processing Group). 2014. Sea-viewing Wide Field-of-view Sensor (SeaWiFS) Ocean Color Data, NASA OB.DAAC, Greenbelt, MD, USA. http://doi. org/10.5067/ORBVIEW-2/SEAWIFS_OC.2014.0 [Consultado en Abril 2020].

Olson, D.M., E. Dinerstein, E.D. Wikramanayake, N.D. Burgess, G.V.N. Powell, E.C. Underwood, J.A. D'Amico, I. Itoua, H.E. 
Strand, J.C. Morrison, C.J. Loucks, T.F. Allnutt, T.H. Ricketts, Y. Kura, J.F. Lamoreux, W.W. Wettengel, P. Hedao \& K.R. Kassem. 2001. Terrestrial ecoregions of the world: a new map of life on Earth. Bioscience 51:933-938.

Pliscoff, P., F. Luebert, H.H. Hilger \& A. Guisan. 2014. Effects of alternative sets of climatic predictors on species distribution models and associated estimates of extinction risk: A test with plants in an arid environment. Ecological Modelling 288:166-177.

Radeloff, V.C., M. Dubinin, N.C. Coops, A.M. Allen, T.M. Brooks, M.K. Clayton, G.C. Costa, C.H. Graham, D.P. Helmers, A.R. Ives, D. Kolesov, A.M. Pidgeon, G. Rapacciuolo, E. Razenkova, N. Suttidate, B.E. Young, L. Zhu \& M.H. Hobi. 2019. The Dynamic Habitat Indices (DHIs) from MODIS and global biodiversity. Remote Sensing of Environment 222:204-214.

Radeloff, V.C., R. B. Hammer, S.I. Stewart, J.S. Fried, S.S. Holcomb \& J.F. McKeefry. 2005. The Wildland-Urban Interface in the United States. Ecological Applications 15:799-805.

Radeloff, V.C., D.P. Helmers, H.A. Kramer, M.H. Mockrin, P.M. Alexandre, A. Bar-Massada, V. Butsic, T.J. Hawbaker, S. Martinuzzi, A.D. Syphard \& S.I. Stewart. 2018. Rapid growth of the U.S. Wildland Urban Interface raises wildfire risk. Proceedings of the National Academy of Sciences 115:3314-3319.

Radeloff, V.C., E. Nelson, A.J. Plantinga, D.J. Lewis, D. Helmers, J.J. Lawler, J.C. Withey, F. Beaudry, S. Martinuzzi, V. Butsic, E. Lonsdorf, D. White \& S. Polasky. 2012. Economic-based projections of future land use in the conterminous United States under alternative policy scenarios. Ecological Applications 22:1036-1049.

Randerson, J.T., G.R. van der Werf, L. Giglio, G.J. Collatz \& P.S. Kasibhatla. 2018. Global Fire Emissions Database, Version 4, (GFEDv4). ORNL DAAC, Oak Ridge, Tennessee, USA.

Robinson, N., J. Regetz \& R.P. Guralnick. 2014. EarthEnv-DEM90: A nearly-global, void-free, multi-scale smoothed, $90 \mathrm{~m}$ digital elevation model from fused ASTER and SRTM data. ISPRS Journal of Photogrammetry and Remote Sensing 87:57-67.

Rodríguez, J.P., L. Brotons, J. Bustamante \& J. Seoane. 2007. The application of predictive modelling of species distribution to biodiversity conservation. Diversity and Distributions 13:243251.
Sauzede R., H.C. Bittig, H. Claustre, O. Pasqueron de Fommervault, J.P. Gattuso, L. Legendre \& K.S. Johnson. 2017. Estimates of Water-Column Nutrient Concentrations and Carbonate System Parameters in the Global Ocean: A novel Approach Based on Neural Networks. Frontiers in Marine Science 4:128.

Sayre, R., S. Noble, S. Hamann, R. Smith, D. Wright, S. Breyer, K. Butler, K. Van Graafeiland, C. Frye, D. Karagulle, D. Hopkins, D. Stephens, K. Kelly, Z. Basher, D. Burton, J. Cress, K. Atkins, D.P. Van Sistine, B. Friesen, R. Alle, T. Allen, P. Aniello, I. Asaad, M.J. Costello, K. Goodin, P. Harris, M. Kavanaugh, H. Lillis, E. Manca, F. Muller-Karger, B. Nyberg, R. Parsons, J. Saarinen, J. Steiner \& A. Reed. 2019. A new 30 meter resolution global shoreline vector and associated global islands database for the development of standardized ecological coastal units. Journal of Operational Oceanography 12:s47-s56.

Sbrocco, E.J. 2014. Paleo-MARSPEC: gridded ocean climate layers for the Mid-Holocene and Last Glacial Maximum. Ecology 95:170.

Sbrocco, E.J. \& P.H. Barber. 2013. MARSPEC: ocean climate layers for marine spatial ecology. Ecology 94:979.

Schepaschenko, D. , J. Chave, O. Phillips, S. Lewis, S. Davies, M. RéjouMéchain, P. Sist, K. Scipal, C. Perger, B. Hérault, N. Labrière, F. Hofhansl, K. Affum-Baffoe, A. Aleinikov, A. Alonso, C. Amani, A. Araujo-Murakami, J. Armston, L. Arroyo, N. Ascarrunz, C. Azevedo, T. Baker, R. Balazy, C. Bedeau, N. Berry, A. Bilous, S.Y. Bilous, P. Bissiengou, L. Blanc, K. Bobkova, T. Braslavskaya, R. Brienen, D. Burslem, R. Condit, A. Cuni-Sánchez, D. Danilina, D. del Castillo-Torres, G. Derroire, L. Descroix, E.D. Sotta, M.V.N. d'Oliveira, C. Dresel, T. Erwin, M. Evdokimenko, J. Falck, T. Feldpausch, E. Foli, R. Foster, S. Fritz, A.D. García-Abril, A. Gornov, M. Gornova, E. Gothard-Bassébé, S. Gourlet-Fleury, M. Guedes, K. Hamer, F.H. Susanty, N. Higuchi, E. Coronado, W. Hubau, S. Hubbell, U. Ilstedt, V. Ivanov, M. Kanashiro, A. Karlsson, V. Karminov, T. Killeen, J.C.K. Koffi, M. Konovalova, F. Kraxner, J. Krejza, H. Krisnawati, L. Krivobokov, M. Kuznetsov, I. Lakyda, P. Lakyda, J.C. Licona, R. Lucas, N. Lukina, D. Lussettu, Y. Malhi, J.A. Manzanera, B. Marimon, B.H.M. Junior, R. Vásquez Martínez, O. Martynenko, M. Matsala, R. Matyashuk, L. Mazzei, H. Memiaghe, C. Mendoza, A. Monteagudo Mendoza, O. Moroziuk, L. Mukhortova, S. Musa, D. Nazimova, T. Okuda, L.C. Oliveira, P. Ontikov, A. Osipov, S. Pietsch, M. Playfair, J. Poulsen, V. Radchenko, K. Rodney, A. Rozak, A. Ruschel, E. Rurishauser, L. See, M. Shchepashchenko, N. Shevchenko, A. Shvidenko, M. Silveira, J. Singh, B. Sonké, C. Souza, K. Stereńczak, L. Stonozhenko, M. Sullivan, J. Szatniewska, H. Taedoumg, H. ter Steege, E. Tikhonova, M. Toledo, O. Trefilova, R. Valbuena, 
L. Valenzuela Gamarra, S. Vasiliev, E. Vedrova, S. Verhovets, E. Vidal, N. Vladimirova, J. Vleminckx, V. Vos, F. Vozmitel, W. Wanek, T. West, H. Woell, J. Woods, V. Wortel, T. Yamada, Z.S. Nur Hajar \& I.C. Zo-Bi. 2019. The Forest Observation System, building a global reference dataset for remote sensing of forest biomass. Scientific Data 6:e198.

Sexton, J.O., P. Noojipady, A. Anand, X.P. Song, S. McMahon, C. Huang, M. Feng, S. Channan \& J.R. Townshend. 2015. A model for the propagation of uncertainty from continuous estimates of tree cover to categorical forest cover and change. Remote Sensing of Environment 156:418-425.

Sexton, J.O., X.P. Song, M. Feng, P. Noojipady, A. Anand, C. Huang, D.H. Kim, K.M. Collins, S. Channan, C. DiMiceli \& J.R. Townshend. 2013. Global, 30-m resolution continuous fields of tree cover: Landsat-based rescaling of MODIS vegetation continuous fields with lidar-based estimates of error. International Journal of Digital Earth 6:427-448.

Song, D.X., C. Huaung, J.O. Sexton, S. Channan, M. Feng \& J.R. Townshend. 2015. Use of Landsat and Corona data for mapping forest cover change from the mid-1960s to 2000s: Case studies from the Eastern United State and Central Brazil. ISPRS Journal of Photogrammetry and Remote Sensing 103:81-92.

Song, X.P., M.C. Hansen, S.V., Stehman, P.V. Potapov, A. Tyukavina, E.F. Vermote \& J.R. Townshend. 2018. Global land change from 1982 to 2016. Nature 560:639-643.

Stewart, S.I., V.C. Radeloff, R.B. Hammer \& T.J. Hawbaker. 2007. Defining the Wildland Urban Interface. Journal of Forestry 105:201-207.

Thornton, P.E., M.M. Thornton, B.W. Mayer, Y. Wei, R. Devarakonda, R.S. Vose \& R.B. Cook. 2016a. Daymet: Daily Surface Weather Data on a $1-\mathrm{km}$ Grid for North America, Version 3. ORNL DAAC, Oak Ridge, Tennessee, USA. https://doi.org/10.3334/ ORNLDAAC/1328 [Consultado en Abril 2020].

Thornton, M.M., P.E. Thornton, Y. Wei, B.W. Mayer, R.B. Cook \& R.S. Vose. 2016b. Daymet: Annual Climate Summaries on a $1-\mathrm{km}$ Grid for North America, Version 3. ORNL DAAC, Oak Ridge, Tennessee, USA. https://doi.org/10.3334/ORNLDAAC/1343 [Consultado en Abril 2020].

Thornton, M.M., P.E. Thornton, Y. Wei, B.W. Mayer, R.B. Cook \& R.S. Vose. 2016c. Daymet: Monthly Climate Summaries on a 1-km Grid for North America, Version 3. ORNL DAAC, Oak
Ridge, Tennessee, USA. https://doi.org/10.3334/ORNLDAAC/1345 [Consultado en Abril 2020].

Title, P.O. \& J.B. Bemmels. 2018. ENVIREM: an expanded set of bioclimatic and topographic variables increases flexibility and improves performance of ecological niche modeling. Ecography 41:291-307.

Trabucco, A. \& R. Zomer. 2019a. Global Aridity Index and Potential Evapotranspiration (ETo) Climate Database v2. figshare.Fileset. https://doi.org/10.6084/m9.figshare.7504448.v3 [Consultado en Abril 2020].

Trabucco, A. \& R.J. Zomer. 2019b. Global High-Resolution SoilWater Balance. figshare. Fileset. https://doi.org/10.6084/ m9.figshare.7707605.v3 [Consultado en Abril 2020].

Tuanmu, M.N. \& W. Jetz. 2015. A global, remote sensing-based characterization of terrestrial habitat heterogeneity for biodiversity and ecosystem modeling. Global Ecology and Biogeography 24:1329-1339.

Tuanmu, M.N. \& W. Jetz. 2014. A global $1 \mathrm{~km}$ consensus land-cover product for biodiversity and ecosystem modeling. Global Ecology and Biogeography 23:1031-1045.

Tyberghein L., H. Verbruggen, K. Pauly, C. Troupin, F. Mineur \& O. De Clerck. 2012. Bio-ORACLE: A global environmental dataset for marine species distribution modelling. Global Ecology and Biogeography 21:272-281.

USGS (United States Geological Survey). 2020. EarthExplorer https://earthexplorer.usgs.gov/ [Consultado en Abril 2020].

van Vliet, J. \& P.H. Verburg. 2017. A short Presentation of CLUMondo. Pp. 485-492. En M.T.C. Olmedo, M. Paegelow, J.F. Mas \& F. Escobar (Eds.), Geomatic approaches for modeling land changes scenarios. Springer, Cham, Suiza.

Vega, G.C., L.R. Pertierra \& M.A. Olalla-Tárraga. 2017. MERRAclim, a high-resolution global dataset of remotely sensed bioclimatic variables for ecological modelling. Scientific Data 4:170078.

Vega, G.C., L.R. Pertierra \& M.A. Olalla-Tárraga. 2018. Data from: MERRAclim, a high-resolution global dataset of remotely sensed bioclimatic variables for ecological modelling, v2, Dryad. https:// doi.org/10.5061/dryad.s2v81 [Consultado en Abril 2020]. 
Venter, O., E.W. Sanderson, A. Magrach, J.R. Allan, J. Beher, K.R. Jones, H.P. Possingham, W.F. Laurance, P. Wood, B.M. Fekete, M.A. Levy \& J.E.M. Watson. 2016a. Data from: Global terrestrial Human Footprint maps for 1993 and 2009, v2, Dryad. https:/doi. org/10.5061/dryad.052q5 [Consultado en Abril 2020].

Venter, O., E.W. Sanderson, A. Magrach, J.R. Allan, J. Beher, K.R. Jones, H.P. Possingham, W.F. Laurance, P. Wood, B.M. Fekete, M.A. Levy \& J.E.M. Watson. 2016b. Data from: Global terrestrial Human Footprint maps for 1993 and 2009. Scientific Data 3:160067.

Verdin, K.L., F.G. Hall, G.J. Collatz, B.W. Meeson, S.O. Los, E. Brown De Colstoun \& D. R. Landis. 2011. ISLSCP II HYDRO1k Elevationderived Products. ORNL DAAC, Oak Ridge, Tennessee, USA. https://doi.org/10.3334/ORNLDAAC/1007 [Consultado en Abril 2020].

Wang, T., A. Hamann, D. Spittlehouse \& C. Carroll. 2016. Locally Downscaled and Spatially Customizable Climate Data for Historical and Future Periods for North America. PLoS One 11:e0156720.

Wilson, A.M. \& W. Jetz. 2016. Remotely Sensed High-Resolution Global Cloud Dynamics for Predicting Ecosystem and Biodiversity Distributions. PLoS Biology 14:e1002415.

Wohner, C., J. Peterseil, D. Poursanidis, T. Kliment, M. Wilson, M. Mirtl \& N. Chrysoulakis. 2019. DEIMS-SDR-A web portal to document research sites and their associated data. Ecological Informatics 51:15-24.
Zhou, Y., A.C.G. Varquez \& M. Kanda. 2019. High-resolution global urban growth projection based on multiple applications of the SLEUTH urban growth model. Scientific Data 6:34.

Zhu, L., V.C. Radeloff, \& A.R. Ives. 2017. Characterizing global patterns of frozen ground with and without snow cover using microwave and MODIS satellite data products. Remote Sensing of Environment 191:168-178.

Zhu L., A.R. Ives, C. Zhang, Y. Guo \& V.C. Radeloff. 2019. Climate change causes functionally colder winters for snow coverdependent organisms. Nature Climate Change 9:886-893.

Zomer, R.J., A. Trabucco, O. van Straaten \& D.A. Bossio. 2006. Carbon, Land and Water: hydrologic dimensions of climate change mitigation through afforestation and reforestation. International Water Management Institute (IWMI) Research Report 101. Colombo, Sri Lanka.

Zomer, R.J., A. Trabucco, D.A. Bossio \& L.V. Verchot. 2008. Climate change mitigation: A spatial analysis of global land suitability for clean development mechanism afforestation and reforestation. Agriculture, Ecosystems and Environment 126:67-80.

Zweng, M.M., J.R. Reagan, D. Seidov, T.P. Boyer, R.A. Locarnini, H.E. Garcia, A.V. Mishonov, O.K. Baranova, K. Weathers, C.R. Paver \& I. Smolyar. 2018. World Ocean Atlas 2018, Volume 2: Salinity. NOAA Atlas NESDIS 82, Silver Spring, Maryland, USA. 